\title{
A POSTERIORI ERROR ESTIMATES FOR NONLINEAR PROBLEMS. FINITE ELEMENT DISCRETIZATIONS OF ELLIPTIC EQUATIONS
}

\author{
R. VERFÜRTH
}

\begin{abstract}
We give a general framework for deriving a posteriori error estimates for approximate solutions of nonlinear problems. In a first step it is proven that the error of the approximate solution can be bounded from above and from below by an appropriate norm of its residual. In a second step this norm of the residual is bounded from above and from below by a similar norm of a suitable finite-dimensional approximation of the residual. This quantity can easily be evaluated, and for many practical applications sharp explicit upper and lower bounds are readily obtained. The general results are then applied to finite element discretizations of scalar quasi-linear elliptic partial differential equations of 2 nd order, the eigenvalue problem for scalar linear elliptic operators of 2 nd order, and the stationary incompressible Navier-Stokes equations. They immediately yield a posteriori error estimates, which can easily be computed from the given data of the problem and the computed numerical solution and which give global upper and local lower bounds on the error of the numerical solution.
\end{abstract}

\section{INTRODUCTION}

The efficiency of a numerical method for the solution of partial differential equations strongly depends on the choice of an "optimal" discretization, the use of a fast and efficient algorithm for the solution of the discrete problem, and a simple, but reliable method for judging the quality of the numerical solution obtained. These three objectives are often interdependent. The first and last one are related to the problem of a posteriori error estimation, i.e., of extracting from the given data of the problem and the computed numerical solution reliable bounds on the error of the numerical solution. Of course, the computation of the a posteriori error estimates should be much less costly than the solution of the original discrete problem.

Within the framework of finite element methods various strategies of a posteriori error estimation have been devised during the last 15-20 years (cf., e.g., $[2,3,20,27]$ and the literature cited there). They can roughly be classified as follows:

(1) residual estimates: Estimate the error of the computed numerical solution by a suitable norm of its residual with respect to the strong form of the differential equation (cf., e.g., [4, 5, 9, 19, 21, 25, 27]).

(2) solution of local problems: Solve locally discrete problems similar to,

Received by the editor December 14, 1992.

1991 Mathematics Subject Classification. Primary 65N30, 65N15, 65J15. 
but simpler than, the original problem and use appropriate norms of the local solutions for error estimation (cf., e.g., [7, 8, 18, 22, 25, 27]).

(3) sharp a priori error estimates: Derive sharp a priori error estimates and use suitable higher-order difference quotients of the computed numerical solution to estimate the higher-order derivatives appearing in the a priori error estimates (cf., e.g., $[15,16])$.

(4) averaging methods: Use some local averaging technique for error estimation (cf., e.g., [6, 21, 29, 30]).

For a certain class of problems and discretizations it was proven in [28] that the methods (1) and (2) are equivalent in the sense that, up to multiplicative constants, they yield the same upper and lower bounds on the error of the numerical solution (cf. also [6, 13, 21] for the comparison of different error estimators). In this context it should be noted that, in order to be efficient, an a posteriori error estimation should yield upper and lower bounds on the error. Clearly, upper bounds are sufficient to ensure that the numerical solution achieves a prescribed tolerance. Lower bounds, however, are essential to guarantee that the error is not overestimated and that its local distribution is correctly resolved. Often, only upper bounds are established in the literature.

Various methods are used for constructing a posteriori error estimators and for proving that they yield upper and/or lower bounds on the error. These methods often depend on a particular class of problems and discretizations. A close inspection, however, reveals that they have certain principles in common. It is the aim of this paper to give a rather general framework that allows one to construct a posteriori error estimators and to prove that they yield upper and lower bounds on the error. In this general context we are satisfied with proving that the upper and lower bounds differ by a multiplicative constant which is independent of the mesh size. We neither intend to derive optimal estimates for this constant nor to prove efficiency of the error estimators, i.e., that the ratio of the true and the estimated error asymptotically tends to 1 . This latter question is addressed for linear problems in e.g. $[2,3,4,5,6,7,13,14]$.

We consider in $\S \S 2-4$ nonlinear equations of the form

$$
F(u)=0
$$

and corresponding discretizations of the form

$$
F_{h}\left(\stackrel{\circ}{u}_{h}\right)=0 \text {. }
$$

Here, $F \in C^{1}\left(X, Y^{*}\right)$ and $F_{h} \in C\left(X_{h}, Y_{h}^{*}\right), X_{h} \subset X$ and $Y_{h} \subset Y$ are finitedimensional subspaces of the Banach spaces $X$ and $Y$, and $*$ denotes the dual of a Banach space.

If $u_{0} \in X$ is a solution of equation (1.1) such that $D F\left(u_{0}\right)$ is an isomorphism of $X$ onto $Y^{*}$ and $D F$ is Lipschitz continuous at $u_{0}$, we prove in Proposition 2.1 that

$$
\underline{c}\|F(u)\|_{Y^{*}} \leq\left\|u-u_{0}\right\|_{X} \leq \bar{c}\|F(u)\|_{Y^{*}}
$$

holds for all $u$ in a suitable neighborhood of $u_{0}$. The constants $\underline{c}$ and $\bar{c}$ depend on $D F\left(u_{0}\right)$ and $D F\left(u_{0}\right)^{-1}$. The proof of Proposition 2.1 is straightforward. The conditions on $F$ can be weakened considerably (cf. Remark 2.3). Inequality (1.3) is a local result. That means that it can be applied to solutions 
of equation (1.2) only if they are sufficiently close to $u_{0}$, i.e., if the discretization is "sufficiently fine". This is not surprising since we are dealing with general nonlinear problems, which may have a large variety of solutions. If problem (1.1) is linear, i.e., $D F$ is constant, inequality (1.3) of course holds for all $u \in X$.

In $\S 3$ we briefly outline how the results of $\S 2$ can be extended to branches of solutions of equation (1.1), including singular points such as simple limit and bifurcation points. The generalization to the case of a regular branch of solutions, i.e., situations covered by the implicit function theorem, is straightforward. The case of a simple limit or bifurcation point can be reduced as in [12] to the case of a regular branch of solutions by suitably blowing up the spaces $X$ and $Y$ and modifying the function $F$. For practical applications it is important that the additional spaces are finite-dimensional. Thus, the cost for evaluating the residual of the modified function is essentially determined by the cost for evaluating the residual of $F$.

In $\S 4$, we estimate the residual $\left\|F\left(u_{h}\right)\right\|_{Y^{*}}$, where $u_{h}$ is an approximate solution of equation (1.2). To this end, we introduce a restriction operator $R_{h}: Y \rightarrow Y_{h}$, a finite-dimensional subspace $\widetilde{Y}_{h} \subset Y$, and an approximation $\widetilde{F}_{h}: X_{h} \rightarrow Y^{*}$ of $F$ at $u_{h}$ which are coupled via inequality (4.1). For practical applications, the construction of $R_{h}$ and $\widetilde{F}_{h}$ is rather straightforward. Usually, $\widetilde{F}_{h}\left(u_{h}\right)$ is obtained by locally projecting $F\left(u_{h}\right)$ onto suitable finite-dimensional spaces. This corresponds to the well-known technique of locally freezing the coefficients of a differential operator. The choice of $\widetilde{Y}_{h}$ on the other hand is less obvious. It is, however, considerably simplified by the auxiliary results of $\S 5$ (see also below). We then prove in Proposition 4.1 that, up to multiplicative constants and additive correction terms, $\left\|F\left(u_{h}\right)\right\|_{Y^{*}}$ is bounded from below and from above by $\left\|\widetilde{F}_{h}\left(u_{h}\right)\right\|_{\widetilde{Y}_{h}^{*}}$. The latter can be evaluated quite easily since its computation is equivalent to a finite-dimensional maximization problem. Moreover, sharp explicit bounds on $\left\|\widetilde{F}_{h}\left(u_{h}\right)\right\|_{\widetilde{Y}_{h}^{*}}$ are readily obtained for many practical applications. When applying the general results to finite element methods, the aforementioned multiplicative constants essentially depend on the element geometry and on the polynomial degree of the finite element functions. In principle, they can be estimated explicitly. The aforementioned correction terms consist of the following quantities:

(1) the residual $\left\|F_{h}\left(u_{h}\right)\right\|_{Y_{n}^{*}}$ of the discrete problem (1.2),

(2) the consistency error $\left\|F\left(u_{h}\right)-F_{h}\left(u_{h}\right)\right\|_{Y_{h}^{*}}$ of the discretization, and

(3) a term which measures the quality of the approximation of $F\left(u_{h}\right)$ by $\widetilde{F}_{h}\left(u_{h}\right)$.

The first quantity can easily be estimated from $u_{h}$ and the given data. The second one can be bounded a priori. For many practical applications one can finally prove that the third quantity is a higher-order perturbation when compared with $\left\|\widetilde{F}_{h}\left(u_{h}\right)\right\|_{\widetilde{Y}_{h}^{*}}$.

In this section we also give a framework which covers some of the a posteriori error estimators based on the solution of auxiliary local problems, such as the one described in $[4,5]$, and which shows that these estimators are equivalent to the residual a posteriori error estimator considered before. 
As already mentioned, we establish in $\S 5$ some auxiliary results which simplify the construction of $\tilde{Y}_{h}$. The main result is of the form (cf. Lemma 5.1)

$$
0 \leq \alpha \leq \inf _{u \in V_{S} \backslash\{0\}} \sup _{v \in V_{S} \backslash\{0\}} \frac{\int_{S} u \psi_{S} v}{\|u\|_{L^{p}(S)}\|v\|_{L^{q}(S)}} \leq 1 .
$$

Here, $1 \leq p \leq \infty, \frac{1}{p}+\frac{1}{q}=1, S$ is either a simplex in $\mathbb{R}^{n}$ or a face of such a simplex, $V_{S}$ is a finite-dimensional space of functions defined on $S$, and $\psi_{S}$ is a cutoff function. It is important to note that the constant $\alpha$ is independent of $S$. Lemma 5.1 is a generalization of Lemma 4.1 in [28]. Thanks to inequality (1.4), one can show that for finite element methods, $\widetilde{Y}_{h}$ can be chosen as the space of all linear combinations of functions $\psi_{S} v$, where $v \in V_{S}$ and $S$ varies through all elements and their faces.

In $\S \S 6-8$ we apply the general results of the previous sections to finite element approximations of scalar quasi-linear elliptic partial differential equations of 2 nd order, the eigenvalue problem for scalar linear elliptic differential operators of 2 nd order, and the stationary incompressible Navier-Stokes equations (cf. Propositions 6.1, 6.3, 6.4, 6.5, 7.1, 8.1, and 8.4). In all examples we obtain upper and lower bounds for the finite element error in terms of a residual a posteriori error estimator. This error estimator essentially consists of the elementwise error of the finite element functions with respect to the strong form of the differential equation and of jumps across inter-element boundaries of that boundary operator which naturally links the strong and weak forms of the differential equation. Some of the results of $\S \S 6-8$ are completely new, others are generalizations of, and improvements upon, results previously obtained in $[4,5,7,8,9,19,25,27,28]$.

\section{ERROR ESTIMATES FOR ISOLATED SOLUTIONS}

Let $X, Y$ be two Banach spaces with norms $\|\cdot\|_{X}$ and $\|\cdot\|_{Y}$. For any element $u \in X$ and any real number $R>0$ set $B(u, R):=\left\{v \in X:\|u-v\|_{X}<R\right\}$. We denote by $\mathscr{L}(X, Y)$ and $\operatorname{Isom}(X, Y) \subset \mathscr{L}(X, Y)$ the Banach space of continuous linear maps of $X$ in $Y$ equipped with the operator norm $\|\cdot\|_{\mathscr{L}(X, Y)}$, and the open subset of linear homeomorphisms of $X$ onto $Y$. By $Y^{*}:=\mathscr{L}(Y, \mathbb{R})$ and $\langle\cdot, \cdot\rangle$ we denote the dual space of $Y$ and the corresponding duality pairing. Finally, $A^{*} \in \mathscr{L}\left(Y^{*}, Y^{*}\right)$ denotes the adjoint of a given linear operator $A \in \mathscr{L}(Y, Y)$.

Let $F \in C^{1}\left(X, Y^{*}\right)$ be a given continuously differentiable function. The following proposition yields a posteriori error estimates for elements in a neighborhood of a solution of equation (1.1).

Proposition 2.1. Let $u_{0} \in X$ be a regular solution of equation (1.1); i.e., $D F\left(u_{0}\right)$ $\in \operatorname{Isom}\left(X, Y^{*}\right)$. Assume that $D F$ is Lipschitz continuous at $u_{0}$; i.e., there is an $R_{0}>0$ such that

$$
\gamma:=\sup _{u \in B\left(u_{0}, R_{0}\right)} \frac{\left\|D F(u)-D F\left(u_{0}\right)\right\|_{\mathscr{L}\left(X, Y^{*}\right)}}{\left\|u-u_{0}\right\|_{X}}<\infty .
$$

Set

$$
R:=\min \left\{R_{0}, \gamma^{-1}\left\|D F\left(u_{0}\right)^{-1}\right\|_{\mathscr{L}\left(Y^{*}, X\right)}^{-1}, 2 \gamma^{-1}\left\|D F\left(u_{0}\right)\right\|_{\mathscr{L}\left(X, Y^{*}\right)}\right\}
$$


Then the following error estimates hold for all $u \in B\left(u_{0}, R\right)$ :

$$
\begin{aligned}
& \frac{1}{2}\left\|D F\left(u_{0}\right)\right\|_{\mathscr{L}\left(X, Y^{*}\right)}^{-1}\|F(u)\|_{Y^{*}} \\
& \quad \leq\left\|u-u_{0}\right\|_{X} \leq 2\left\|D F\left(u_{0}\right)^{-1}\right\|_{\mathscr{L}\left(Y^{*}, X\right)}\|F(u)\|_{Y^{*}}
\end{aligned}
$$

Proof. Let $u \in B\left(u_{0}, R\right)$. We then have

$$
u-u_{0}=D F\left(u_{0}\right)^{-1}\left\{F(u)+\int_{0}^{1}\left[D F\left(u_{0}\right)-D F\left(u_{0}+t\left(u-u_{0}\right)\right)\right]\left(u-u_{0}\right) d t\right\}
$$

and thus

$$
\begin{aligned}
\| u- & u_{0} \|_{X} \\
\leq & \left\|D F\left(u_{0}\right)^{-1}\right\|_{\mathscr{L}\left(Y^{*}, X\right)} \\
& \cdot\left\{\|F(u)\|_{Y^{*}}+\int_{0}^{1}\left\|D F\left(u_{0}\right)-D F\left(u_{0}+t\left(u-u_{0}\right)\right)\right\|_{\mathscr{L}\left(X, Y^{*}\right)}\left\|u-u_{0}\right\|_{X} d t\right\} \\
\leq & \left\|D F\left(u_{0}\right)^{-1}\right\|_{\mathscr{L}\left(Y^{*}, X\right)}\left\{\|F(u)\|_{Y^{*}}+\frac{1}{2} \gamma\left\|u-u_{0}\right\|_{X}^{2}\right\} \\
\leq & \left\|D F\left(u_{0}\right)^{-1}\right\|_{\mathscr{L}\left(Y^{*}, X\right)}\|F(u)\|_{Y^{*}}+\frac{1}{2}\left\|u-u_{0}\right\|_{X} .
\end{aligned}
$$

This yields the second inequality in (2.1).

On the other hand, we have for all $\varphi \in Y$ with $\|\varphi\|_{Y}=1$

$$
\begin{aligned}
\langle F(u), \varphi\rangle= & \left\langle D F\left(u_{0}\right)\left(u-u_{0}\right), \varphi\right\rangle \\
& +\left\langle\int_{0}^{1}\left[D F\left(u_{0}+t\left(u-u_{0}\right)\right)-D F\left(u_{0}\right)\right]\left(u-u_{0}\right) d t, \varphi\right\rangle
\end{aligned}
$$

and thus

$$
\begin{aligned}
\|F(u)\|_{Y^{*}} \leq & \left\|D F\left(u_{0}\right)\right\|_{\mathscr{L}\left(X, Y^{*}\right)}\left\|u-u_{0}\right\|_{X} \\
& +\int_{0}^{1}\left\|D F\left(u_{0}+t\left(u-u_{0}\right)\right)-D F\left(u_{0}\right)\right\|_{\mathscr{L}\left(X, Y^{*}\right)}\left\|u-u_{0}\right\|_{X} d t \\
\leq & \left\|D F\left(u_{0}\right)\right\|_{\mathscr{L}\left(X, Y^{*}\right)}\left\|u-u_{0}\right\|_{X}+\frac{1}{2} \gamma\left\|u-u_{0}\right\|_{X}^{2} \\
\leq & 2\left\|D F\left(u_{0}\right)\right\|_{\mathscr{L}\left(X, Y^{*}\right)}\left\|u-u_{0}\right\|_{X} .
\end{aligned}
$$

This proves the first inequality in (2.1).

Remark 2.2. In the examples of $\S \S 6-8, X$ and $Y$ are closed subspaces of suitable Sobolev spaces of functions defined on an open set $\Omega \subset \mathbb{R}^{n}$. When considering in equation (2.2) only functions $\varphi$ with support in a given open subset $\omega \subset \Omega$, one then often obtains lower bounds for $u-u_{0}$ restricted to $\omega$.

Remark 2.3. The conditions about $F$ can be weakened. Assume, e.g., that $F \in C\left(X, Y^{*}\right), F\left(u_{0}\right)=0$, and that there are an $R>0$ and two monotonically increasing homeomorphisms $\varrho, \sigma$ of $[0, \infty)$ onto itself such that

$$
\varrho\left(\left\|u-u_{0}\right\|_{X}\right) \leq\|F(u)\|_{Y^{*}} \leq \sigma\left(\left\|u-u_{0}\right\|_{X}\right) \quad \forall u \in B\left(u_{0}, R\right) .
$$


We then trivially have

$$
\sigma^{-1}\left(\|F(u)\|_{Y^{*}}\right) \leq\left\|u-u_{0}\right\|_{X} \leq \varrho^{-1}\left(\|F(u)\|_{Y^{*}}\right) \quad \forall u \in B\left(u_{0}, R\right) .
$$

The first inequality in (2.3) is satisfied if, e.g., $F$ is strongly monotone in a neighborhood of $u_{0}$. The second inequality in (2.3) holds if, e.g., $F$ is Hölder continuous at $u_{0}$.

\section{ERROR ESTIMATES FOR BRANCHES OF SOLUTIONS}

In this section we briefly outline how the results of the previous section may be extended to branches of solutions of equation (1.1), including simple limit and bifurcation points. To this end, we assume that $X=\mathbb{R}^{m} \times V, m \geq 1$, and that $u_{0}=\left(\lambda_{0}, v_{0}\right)$ is a solution of equation (1.1).

We first consider the case that $u_{0}$ is a regular point, i.e.,

$$
D_{V} F\left(u_{0}\right) \in \operatorname{Isom}\left(V, Y^{*}\right) \text {. }
$$

The implicit function theorem then implies that there are neighborhoods $I$ of $\lambda_{0}$ in $\mathbb{R}^{m}$ and $U$ of $v_{0}$ in $V$ and a continuous map $\lambda \rightarrow v_{\lambda}$ from $I$ into $U$ such that $v_{\lambda_{0}}=v_{0}$ and every $u_{\lambda}:=\left(\lambda, v_{\lambda}\right)$ is a solution of equation (1.1) with $D_{V} F\left(u_{\lambda}\right) \in \operatorname{Isom}\left(V, Y^{*}\right)$. Assume that there is an $R_{0}^{*}>0$ such that

$$
\gamma^{*}:=\sup _{\lambda \in I} \sup _{v \in B\left(v_{\lambda}, R_{0}^{*}\right)} \frac{\left\|D_{V} F(\lambda, v)-D_{V} F\left(\lambda, v_{\lambda}\right)\right\|_{\mathscr{L}\left(V, Y^{*}\right)}}{\left\|v-v_{\lambda}\right\|_{V}}<\infty,
$$

and set

$$
R^{*}:=\min \left\{R_{0}^{*}, \gamma^{*^{-1}} \sup _{\lambda \in I}\left\|D_{V} F\left(u_{\lambda}\right)^{-1}\right\|_{\mathscr{L}\left(Y^{*}, V\right)}^{-1}, 2{\gamma^{*}}^{-1} \sup _{\lambda \in I}\left\|D_{V} F\left(u_{\lambda}\right)\right\|_{\mathscr{L}\left(V, Y^{*}\right)}\right\}
$$

With the same arguments as in the proof of Proposition 2.1 we then obtain for all $\lambda \in I$ and all $v \in B\left(v_{\lambda}, R^{*}\right)$ the estimates

$$
\begin{aligned}
& \frac{1}{2}\left\|D_{V} F\left(u_{\lambda}\right)\right\|_{\mathscr{L}\left(V, Y^{*}\right)}^{-1}\|F(\lambda, v)\|_{Y^{*}} \\
& \quad \leq\left\|v-v_{\lambda}\right\|_{V} \leq 2\left\|D_{V} F\left(u_{\lambda}\right)^{-1}\right\|_{\mathscr{L}\left(Y^{*}, V\right)}\|F(\lambda, v)\|_{Y^{*}} .
\end{aligned}
$$

As described in [12], the case where $u_{0}$ is not a regular point, but a simple limit or bifurcation point, may be reduced to the case of a regular point by suitably blowing up the spaces $X$ and $Y$ and modifying the function $F$. For completeness, we briefly describe this procedure.

Consider first the case that $u_{0}$ is a simple limit point; i.e., $D F\left(u_{0}\right)$ is a Fredholm operator of $X$ onto $Y^{*}$ with index $m$ and $\operatorname{Range}\left(D F\left(u_{0}\right)\right)=Y^{*}$ but $D_{V} F\left(u_{0}\right) \notin \operatorname{Isom}\left(V, Y^{*}\right)$. Choose a linear operator $B \in \mathscr{L}\left(X, \mathbb{R}^{m}\right)$ with $\operatorname{ker}(B) \cap \operatorname{ker}\left(D F\left(u_{0}\right)\right)=\{0\}$ and define $\Phi \in C^{1}\left(\mathbb{R}^{m} \times X, \mathbb{R}^{m} \times Y^{*}\right)$ by

$$
\Phi(t, u):=\left(B\left(u-u_{0}\right)-t, F(u)\right) \text {. }
$$

Then, $\left(0, u_{0}\right)$ is a regular point of $\Phi$ (with respect to the parameter $t$ ), and we are back to the situation described in the first part of this section. Since $B$ is linear, conditions about the Lipschitz continuity of $D \Phi$ reduce to those on $D F$. Equation (3.1) yields in this case estimates of the form

$$
\begin{aligned}
\underline{c}\left\{\left\|B\left(u-u_{0}\right)-t\right\|_{\mathbb{R}^{m}}+\|F(u)\|_{Y^{*}}\right\} & \leq\left\|\lambda-\lambda_{t}\right\|_{\mathbb{R}^{m}}+\left\|v-v_{t}\right\|_{V} \\
& \leq \bar{c}\left\{\left\|B\left(u-u_{0}\right)-t\right\|_{\mathbb{R}^{m}}+\|F(u)\|_{Y^{*}}\right\}
\end{aligned}
$$


for all $t$ in a suitable neighborhood of 0 and all $u=(\lambda, v)$ in a suitable neighborhood of $u_{t}=\left(\lambda_{t}, v_{t}\right)$. Here, $t \rightarrow u_{t}$ is a regular branch of solutions of $\Phi(t, u)=0$. Note, that $B u_{0}$ is often known explicitly and that the estimation of $\left\|B\left(u-u_{0}\right)-t\right\|_{\mathbb{R}^{m}}$ is straightforward, since it is a low-dimensional maximization problem. The term $\|F(u)\|_{Y^{*}}$, on the other hand, may be estimated by the methods of the next section, as in the case of regular solutions.

Next, we consider the case of a simple bifurcation from the trivial branch. That is, we assume that $u_{0}=\left(\lambda_{0}, 0\right)$ and that $D_{V} F\left(u_{0}\right)$ is a Fredholm operator with index 0 and $\operatorname{dim} \operatorname{ker}\left(D_{V} F\left(u_{0}\right)\right)=1$. Choose a $w_{0} \in \operatorname{ker}\left(D_{V} F\left(u_{0}\right)\right) \backslash\{0\}$ and a linear functional $l \in \mathscr{L}(V, \mathbb{R})$ with $l\left(w_{0}\right)=1$. Define the function $\Phi \in C\left(\mathbb{R} \times X, \mathbb{R} \times Y^{*}\right)$ by

$$
\Phi(t, u):= \begin{cases}\left(l(v)-1, \frac{1}{t} F(\lambda, t v)\right), & t \neq 0, u=(\lambda, v) \in X, \\ \left(l(v)-1, D_{V} F(\lambda, 0) v\right), & t=0, u=(\lambda, v) \in X .\end{cases}
$$

Conditions about the Lipschitz continuity of $D \Phi$ now reduce to those on $D^{2} F$. Obviously, we have $\Phi\left(0, \tilde{u}_{0}\right)=0$, where $\tilde{u}_{0}:=\left(\lambda_{0}, w_{0}\right)$. If $F$ is of class $C^{2}$ in a neighborhood of $u_{0}$ and $D_{\lambda v}^{2} F\left(u_{0}\right) w_{0} \notin$ Range $D_{V} F\left(u_{0}\right)$, we conclude that $\tilde{u}_{0}$ is a regular point, and we are once more back to the situation described in the first part of this section. Equation (3.1) now yields estimates of the form

$$
\begin{aligned}
\underline{c}\left\{|l(w)-1|+\left\|D_{V} F(\lambda, 0) w\right\|_{Y^{*}}\right\} & \leq\left\|\lambda-\lambda_{0}\right\|_{\mathbb{R}^{m}}+\left\|w-w_{0}\right\|_{V} \\
& \leq \bar{c}\left\{|l(w)-1|+\left\|D_{V} F(\lambda, 0) w\right\|_{Y^{*}}\right\}
\end{aligned}
$$

for all $(\lambda, w)$ in a suitable neighborhood of $\tilde{u}_{0}$ and

$$
\begin{aligned}
\underline{c}\left\{|l(w)-1|+\left\|\frac{1}{t} F(\lambda, t w)\right\|_{Y^{*}}\right\} & \leq\left\|\lambda-\lambda_{t}\right\|_{\mathbb{R}^{m}}+\left\|w-w_{t}\right\|_{V} \\
& \leq \bar{c}\left\{|l(w)-1|+\left\|\frac{1}{t} F(\lambda, t w)\right\|_{Y^{*}}\right\}
\end{aligned}
$$

for all $t \neq 0$ in a neighborhood of 0 and all $(\lambda, w)$ in a suitable neighborhood of $\tilde{u}_{t}=\left(\lambda_{t}, w_{t}\right)$. Here, $t \rightarrow \tilde{u}_{t}$ is a regular branch of solutions of $\Phi(t, u)=$ 0 . Note that the constants in equations (3.3), (3.4) now depend on second derivatives of $F$.

Finally, we consider the case of a simple bifurcation point; i.e., $D_{V} F\left(u_{0}\right)$ is a Fredholm operator of index 0 and $q:=\operatorname{dim}\left(\operatorname{ker} D F\left(u_{0}\right)\right)-m \geq 1$. Choose a basis $\varphi_{1}^{*}, \ldots, \varphi_{q}^{*}$ of $Y^{*} \backslash \operatorname{Range}\left(D F\left(u_{0}\right)\right)$, set $\widehat{X}:=\mathbb{R}^{q} \times X, \hat{u}_{0}:=\left(0, u_{0}\right)$, and define the function $\widehat{F} \in C^{1}(\widehat{X}, Y)$ by

$$
\widehat{F}(\hat{u}):=F(u)-\sum_{i=1}^{q} f_{i} \varphi_{i}^{*} \quad \forall \hat{u}=(f, u) \in \widehat{X} .
$$

Obviously, we have $\widehat{F}\left(\hat{u}_{0}\right)=0$. Moreover, $D \widehat{F}\left(\hat{u}_{0}\right)$ is a Fredholm operator with index $m+q$ and $\operatorname{Range}\left(D \widehat{F}\left(\hat{u}_{0}\right)\right)=Y^{*}$. Replacing $X, u_{0}$, and $F$ by $\widehat{X}, \hat{u}_{0}$, and $\widehat{F}$, respectively, we are thus back to the situation considered in the second part of this section.

\section{ESTIMATION OF THE RESIDUAL}

Let $X_{h} \subset X$ and $Y_{h} \subset Y$ be finite-dimensional subspaces and $F_{h} \in C\left(X_{h}, Y_{h}^{*}\right)$ be an approximation of $F$. We want to estimate $\left\|F\left(u_{h}\right)\right\|_{Y^{*}}$, where $u_{h} \in X_{h}$ is an approximate solution of equation (1.2). 
In what follows, $c, c_{0}, c_{1}, \ldots$ denote various constants which are independent of $h$.

Proposition 4.1. Let $u_{h} \in X_{h}$ be an approximate solution of equation (1.2); i.e., $\left\|F_{h}\left(u_{h}\right)\right\|_{Y_{h}^{*}}$ is "small". Assume that there are a restriction operator $R_{h} \in$ $\mathscr{L}\left(Y, Y_{h}\right)$, a finite-dimensional subspace $\widetilde{Y}_{h} \subset Y$, and an approximation $\widetilde{F}_{h}$ : $X_{h} \rightarrow Y^{*}$ of $F$ at $u_{h}$ such that

$$
\left\|\left(\operatorname{Id}_{Y}-R_{h}\right)^{*} \widetilde{F}_{h}\left(u_{h}\right)\right\|_{Y^{*}} \leq c_{0}\left\|\widetilde{F}_{h}\left(u_{h}\right)\right\|_{\widetilde{Y}_{h}^{*}}
$$

Then the following estimates hold:

$$
\begin{aligned}
\left\|F\left(u_{h}\right)\right\|_{Y^{*}} \leq & c_{0}\left\|\widetilde{F}_{h}\left(u_{h}\right)\right\|_{\widetilde{Y}_{h}^{*}}+\left\|\left(\operatorname{Id}_{Y}-R_{h}\right)^{*}\left[F\left(u_{h}\right)-\widetilde{F}_{h}\left(u_{h}\right)\right]\right\|_{Y^{*}} \\
& +\left\|R_{h}\right\|_{\mathscr{L}\left(Y, Y_{h}\right)}\left\|F\left(u_{h}\right)-F_{h}\left(u_{h}\right)\right\|_{Y_{h}^{*}} \\
& +\left\|R_{h}\right\|_{\mathscr{L}\left(Y, Y_{h}\right)}\left\|F_{h}\left(u_{h}\right)\right\|_{Y_{h}^{*}}
\end{aligned}
$$

and

$$
\left\|\widetilde{F}_{h}\left(u_{h}\right)\right\|_{\widetilde{Y}_{h}^{*}} \leq\left\|F\left(u_{h}\right)\right\|_{\widetilde{Y}_{h}^{*}}+\left\|F\left(u_{h}\right)-\widetilde{F}_{h}\left(u_{h}\right)\right\|_{\widetilde{Y}_{h}^{*}} .
$$

Remark 4.2. In the examples of $\S \S 6-8, X_{h}$ and $Y_{h}$ are suitable finite element spaces. The choice of $R_{h}$ is then quite natural. $\widetilde{F}_{h}\left(u_{h}\right)$ is obtained by projecting $F\left(u_{h}\right)$ elementwise onto suitable finite-dimensional spaces. This construction is also rather standard. The main difficulty is to find a space $\widetilde{Y}_{h}$ such that inequality (4.1) is satisfied. This task is simplified by the auxiliary results of $\S 5$. The second terms on the right-hand sides of equations (4.2) and (4.3) measure the quality of the approximation $\widetilde{F}_{h}\left(u_{h}\right)$ to $F\left(u_{h}\right)$. Usually, they are higherorder terms when compared with $\left\|\widetilde{F}_{h}\left(u_{h}\right)\right\|_{\widetilde{Y}_{h}^{*}}$. The term $\left\|F\left(u_{h}\right)-F_{h}\left(u_{h}\right)\right\|_{Y_{h}^{*}}$ is the consistency error of the discretization. The term $\left\|F_{h}\left(u_{h}\right)\right\|_{Y_{h}^{*}}$ measures the residual of the algebraic equation (1.2) and can easily be evaluated.

Proof of Proposition 4.1. Consider an arbitrary element $\varphi \in Y$ with $\|\varphi\|_{Y}=1$. We then have

$$
\begin{aligned}
\left\langle F\left(u_{h}\right), \varphi\right\rangle=\langle & \left\langle\widetilde{F}_{h}\left(u_{h}\right), \varphi-R_{h} \varphi\right\rangle+\left\langle F\left(u_{h}\right)-\widetilde{F}_{h}\left(u_{h}\right), \varphi-R_{h} \varphi\right\rangle \\
& +\left\langle F\left(u_{h}\right)-F_{h}\left(u_{h}\right), R_{h} \varphi\right\rangle+\left\langle F_{h}\left(u_{h}\right), R_{h} \varphi\right\rangle \\
\leq & \left\|\left(\operatorname{Id}_{Y}-R_{h}\right)^{*} \widetilde{F}_{h}\left(u_{h}\right)\right\|_{Y^{*}}+\left\|\left(\operatorname{Id}_{Y}-R_{h}\right)^{*}\left[F\left(u_{h}\right)-\widetilde{F}_{h}\left(u_{h}\right)\right]\right\|_{Y^{*}} \\
& +\left\|R_{h}\right\|_{\mathscr{L}\left(Y, Y_{h}\right)}\left\|F\left(u_{h}\right)-F_{h}\left(u_{h}\right)\right\|_{Y_{h}^{*}}+\left\|R_{h}\right\|_{\mathscr{L}\left(Y, Y_{h}\right)}\left\|F_{h}\left(u_{h}\right)\right\|_{Y_{h}^{*}} .
\end{aligned}
$$

Together with inequality (4.1), this proves estimate (4.2). Estimate (4.3) follows from the triangle inequality.

When combining Propositions 2.1 and 4.1 we obtain a residual a posteriori error estimator. The following proposition together with Proposition 2.1 yields a framework for some of those a posteriori error estimators which are based on the solution of auxiliary local problems, such as the one described in $[4,5]$.

Proposition 4.3. Let $u_{h} \in X_{h}$ be an approximate solution of equation (1.2). Assume that there are finite-dimensional subspaces $\widehat{X}_{h} \subset X$ and $\widehat{Y}_{h} \subset Y$ and $a$ linear operator $B \in \operatorname{Isom}\left(\widehat{X}_{h}, \widehat{Y}_{h}^{*}\right)$ such that $\widetilde{Y}_{h} \subset \widehat{Y}_{h}$ and

$$
\left\|\widetilde{F}_{h}\left(u_{h}\right)\right\|_{\widehat{Y}_{h}^{*}} \leq c_{1}\left\|\widetilde{F}_{h}\left(u_{h}\right)\right\|_{\widetilde{Y}_{h}^{*}}
$$


Let $\hat{u}_{h} \in \widehat{X}_{h}$ be the unique solution of

$$
\left\langle B \hat{u}_{h}, \varphi\right\rangle=\left\langle\widetilde{F}_{h}\left(u_{h}\right), \varphi\right\rangle \quad \forall \varphi \in \widehat{Y}_{h} .
$$

Then the following estimates hold:

$$
\|B\|_{\mathscr{L}\left(\widehat{X}_{h}, \widehat{Y}_{h}^{*}\right)}^{-1}\left\|\widetilde{F}_{h}\left(u_{h}\right)\right\|_{\widetilde{Y}_{h}^{*}} \leq\left\|u_{h}\right\|_{\widehat{X}_{h}} \leq c_{1}\left\|B^{-1}\right\|_{\mathscr{L}\left(\widehat{Y}_{h}^{*}, \widehat{X}_{h}\right)}\left\|\widetilde{F}_{h}\left(u_{h}\right)\right\|_{\widetilde{Y}_{h}^{*}} .
$$

Proof. Since $B \in \operatorname{Isom}\left(\widehat{X}_{h}, \widehat{Y}_{h}^{*}\right)$, we immediately obtain from equation (4.5) the estimate

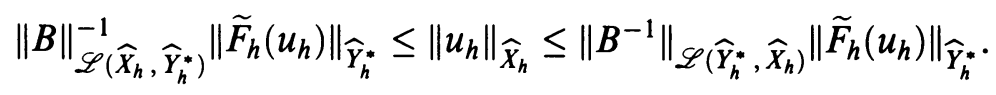

Together with inequality (4.4), this proves the upper bound of inequality (4.6). Since $\tilde{Y}_{h} \subset \widehat{Y}_{h}$, we have

$$
\left\|\widetilde{F}_{h}\left(u_{h}\right)\right\|_{\widetilde{Y}_{h}^{*}} \leq\left\|\widetilde{F}_{h}\left(u_{h}\right)\right\|_{\widehat{Y}_{h}^{*}} .
$$

Together with inequality (4.7), this proves the lower bound of inequality (4.6).

Remark 4.4. Usually, $B$ is some approximation of $D F\left(u_{h}\right)$. The construction of $\widehat{Y}_{h}$ and the proof of inequality (4.4) are similar to the construction of $\widetilde{Y}_{h}$ and the proof of inequality (4.1) and are simplified by the auxiliary results of $\S 5$. Once $B$ and $\widehat{Y}_{h}$ are chosen, the construction of $\widehat{X}_{h}$ is quite obvious from the condition that $B \in \operatorname{Isom}\left(\widehat{X}_{h}, \widehat{Y}_{h}^{*}\right)$.

\section{AuXiliary Results}

Let $\Omega$ be a bounded, connected, open domain in $\mathbb{R}^{n}, n \geq 2$, with polyhedral boundary $\Gamma$. For any open subset $\omega \subset \Omega$ with Lipschitz boundary $\gamma$, we denote by $W^{k, s}(\omega), k \in \mathbb{N}, 1 \leq s \leq \infty, L^{s}(\omega):=W^{0, s}(\omega)$, and $L^{s}(\gamma)$ the usual Sobolev and Lebesgue spaces equipped with the standard norms $\|\cdot\|_{k, s ; \omega}:=\|\cdot\|_{W^{k, s}(\omega)}$ and $\|\cdot\|_{s ; \gamma}:=\|\cdot\|_{L^{s}(\gamma)}$ (cf. [1]). If $\omega=\Omega$, we omit the index $\omega$. We use the same notation for the corresponding norms of vector-valued functions.

Let $\mathscr{T}_{h}, h>0$, be a family of partitions of $\Omega$ into $n$-simplices, which satisfies the following conditions:

(1) Any two simplices in $\mathscr{T}_{h}$ are either disjoint or share a complete smooth submanifold of their boundaries.

(2) The ratio $h_{T} / \varrho_{T}$ is bounded from above independently of $T \in \mathscr{T}_{h}$ and $h>0$.

Here, $h_{T}, \varrho_{T}$, and $h_{E}$ denote the diameter of $T \in \mathscr{T}_{h}$, the diameter of the largest ball inscribed into $T$, and the diameter of a face $E$ of $T$. Note, that condition (2) allows the use of locally refined meshes and that it implies that the ratio $h_{T} / h_{E}$, for all $T \in \mathscr{T}_{h}$ and all faces $E$ of $T$, is bounded from above and from below by constants which are independent of $h, T$, and $E$.

Denote by $\mathscr{E}_{h}$ the set of all faces of all $T \in \mathscr{T}_{h}$. The set $\mathscr{E}_{h}$ may be decomposed as $\mathscr{E}_{h}=\mathscr{E}_{h, \Omega} \cup \mathscr{E}_{h, \Gamma}, \mathscr{E}_{h, \Omega} \cap \mathscr{E}_{h, \Gamma}=\varnothing$, where $\mathscr{E}_{h, \Gamma}$ denotes the set of all faces lying on $\Gamma$. Given an $E \in \mathscr{E}_{h}$, we denote by $\omega_{E}$ the union of all simplices in $\mathscr{T}_{h}$ having $E$ as a face. Similarly, $\omega_{T}, T \in \mathscr{T}_{h}$, is the union of all simplices sharing a face with $T$. For any $E \in \mathscr{E}_{h}$ and any piecewise continuous 
function $\varphi$, we denote by $[\varphi]_{E}$ the jump of $\varphi$ across $E$ in a fixed direction. Here, $\varphi$ is continued by 0 outside $\Omega$ and the direction is given by the exterior normal of $\Gamma$ if $E \in \mathscr{E}_{h, \Gamma}$.

For $k \in \mathbb{N}$, we define

$$
S_{h}^{k,-1}:=\left\{\varphi: \Omega \rightarrow \mathbb{R}:\left.\varphi\right|_{T} \in \Pi_{k} \forall T \in \mathscr{T}_{k}\right\}, \quad S_{h}^{k, 0}:=S_{h}^{k,-1} \cap C(\bar{\Omega}) .
$$

Here, $\Pi_{k}, k \geq 0$, is the space of polynomials of degree at most $k$. Moreover, we denote by $\pi_{k, S}, S \in \mathscr{T}_{h} \cup \mathscr{E}_{h}$, the $L^{2}$-projection of $L^{1}(S)$ onto $\left.\Pi_{k}\right|_{s}$.

Using standard scaling arguments for finite elements, we finally conclude from [11] that there is an "interpolation" operator $I_{h}: L^{1}(\Omega) \rightarrow S_{h}^{1,0}$ which satisfies the following error estimates for all $T \in \mathscr{T}_{h}, E \in \mathscr{E}_{h}$, and $1 \leq q \leq \infty$ :

$$
\begin{gathered}
\left\|\varphi-I_{h} \varphi\right\|_{k, q ; T} \leq c_{1} h_{T}^{l-k}\|\varphi\|_{l, q ; \tilde{\omega}_{T}} \quad \forall 0 \leq k \leq l \leq 1, \varphi \in W^{l, q}\left(\widetilde{\omega}_{T}\right), \\
\left\|\varphi-I_{h} \varphi\right\|_{q ; E} \leq c_{2} h_{E}^{1-1 / q}\|\varphi\|_{1, q ; \tilde{\omega}_{E}} \quad \forall \varphi \in W^{1, q}\left(\widetilde{\omega}_{E}\right),
\end{gathered}
$$

where $\widetilde{\omega}_{T}$ and $\widetilde{\omega}_{E}$ denote the union of all elements having a nonempty intersection with $T$ and $E$, respectively. Here and in what follows, we adopt the usual convention that $1 / \infty:=0$.

Denote by $\hat{T}:=\left\{\hat{x} \in \mathbb{R}^{n}: \sum_{i=1}^{n} \hat{x}_{i} \leq 1, \hat{x}_{j} \geq 0,1 \leq j \leq n\right\}$ the reference $n$-simplex. Set $\widehat{E}:=\widehat{T} \cap\left\{\hat{x} \in \mathbb{R}^{n}: \hat{x}_{n}=0\right\}$, and let $\hat{x}_{\widehat{T}}$ and $\hat{x}_{\widehat{E}}$ be the barycenters of $\widehat{T}$ and $\widehat{E}$, respectively. The following conditions uniquely define two functions $\psi_{\widehat{T}}, \psi_{\widehat{E}} \in C^{\infty}(\widehat{T}, \mathbb{R})$ :

$$
\begin{array}{llll}
\psi_{\widehat{T}} \in \Pi_{n+1}, & \psi_{\widehat{T}}\left(\hat{x}_{\widehat{T}}\right)=1, & \psi_{\widehat{T}}=0 & \text { on } \partial \widehat{T}, \\
\psi_{\widehat{E}} \in \Pi_{n}, & \psi_{\widehat{E}}\left(\hat{x}_{\widehat{E}}\right)=1, & \psi_{\widehat{E}}=0 & \text { on } \partial \widehat{T} \backslash \widehat{E} .
\end{array}
$$

Note, that the above conditions, in particular, imply that

$$
0 \leq \psi_{\widehat{T}} \leq 1, \quad 0 \leq \psi_{\widehat{E}} \leq 1 \quad \text { in } \widehat{T} .
$$

We define a continuation operator $\widehat{P}: L^{\infty}(\widehat{E}) \rightarrow L^{\infty}(\widehat{T})$ by

$$
\widehat{P} \hat{u}\left(\hat{x}_{1}, \ldots, \hat{x}_{n}\right):=\hat{u}\left(\hat{x}_{1}, \ldots, \hat{x}_{n-1}\right) \quad \forall \hat{x} \in \widehat{T}, \hat{u} \in L^{\infty}(\widehat{E}) .
$$

Finally, $V_{\widehat{T}} \subset L^{\infty}(\widehat{T})$ and $V_{\widehat{E}} \subset L^{\infty}(\widehat{E})$ are two arbitrary finite-dimensional spaces, which are kept fixed throughout this section.

Let $T \in \mathscr{T}_{h}$ be an arbitrary $n$-simplex and $E \subset \partial T$ be a face of $T$. There is an invertible affine mapping $F_{T}: \widehat{T} \rightarrow T, \hat{x} \rightarrow x:=F_{T}(\hat{x})=b_{T}+B_{T} \hat{x}$ such that $\widehat{T}$ is mapped onto $T$ and $\widehat{E}$ is mapped onto $E$. Denote by $B_{T}^{\prime}$ the matrix which is obtained from $B_{T}$ by discarding its last column, and set $\beta_{T}:=\operatorname{det}\left(B_{T}^{\prime t} B_{T}^{\prime}\right)^{1 / 2}$, the Gram determinant of the transformation $\widehat{E} \rightarrow E$. Set

$$
\begin{array}{ll}
\psi_{T}:=\psi_{\widehat{T}} \circ F_{T}^{-1}, & \psi_{E}:=\psi_{\widehat{E}} \circ F_{T}^{-1}, \\
V_{T}:=\left\{\hat{u} \circ F_{T}^{-1}: \hat{u} \in V_{\widehat{T}}\right\}, & V_{E}:=\left\{\hat{\sigma} \circ F_{T}^{-1}: \hat{\sigma} \in V_{\widehat{E}}\right\} .
\end{array}
$$

Finally, we define the continuation operator $P: L^{\infty}(E) \rightarrow L^{\infty}(T)$ by

$$
P \sigma:=\left[\widehat{P} \sigma \circ F_{T}\right] \circ F_{T}^{-1} .
$$

In what follows, $p, q$ are two fixed real numbers with $1 \leq p \leq \infty$ and $\frac{1}{p}+\frac{1}{q}=1$, and $\left\||\cdot \||\right.$ denotes the spectral norm on $\mathbb{R}^{n \times n}$. 
Lemma 5.1. There are constants $c_{1}, \ldots, c_{7}$, which only depend on the spaces $V_{\widehat{T}}$ and $V_{\widehat{E}}$, the number $p$, and the ratio $h_{T} \varrho_{T}$, such that the following inequalities hold for all $u \in V_{T}$ and all $\sigma \in V_{E}$ :

$$
\begin{aligned}
c_{1}\|u\|_{0, p ; T} & \leq \sup _{v \in V_{T}} \frac{\int_{T} u \psi_{T} v}{\|v\|_{0, q ; T}} \leq\|u\|_{0, p ; T}, \\
c_{2}\|\sigma\|_{p ; E} & \leq \sup _{\tau \in V_{E}} \frac{\int_{E} \sigma \psi_{E} \tau}{\|\tau\|_{q ; E}} \leq\|\sigma\|_{p ; E}, \\
c_{3} h_{T}^{-1}\left\|\psi_{T} u\right\|_{0, q ; T} & \leq\left\|\nabla\left(\psi_{T} u\right)\right\|_{0, q ; T} \leq c_{4} h_{T}^{-1}\left\|\psi_{T} u\right\|_{0, q ; T}, \\
c_{5} h_{T}^{-1}\left\|\psi_{E} P \sigma\right\|_{0, q ; T} & \leq\left\|\nabla\left(\psi_{E} P \sigma\right)\right\|_{0, q ; T} \leq c_{6} h_{T}^{-1}\left\|\psi_{E} P \sigma\right\|_{0, q ; T}, \\
\left\|\psi_{E} P \sigma\right\|_{0, q ; T} & \leq c_{7} h_{T}^{1 / q}\|\sigma\|_{q ; E} .
\end{aligned}
$$

Proof. The upper bounds of equations (5.3), (5.4) immediately follow from Hölder's inequality and $0 \leq \psi_{T} \leq 1,0 \leq \psi_{E} \leq 1$.

In order to prove the lower bound of equation (5.3), one easily checks that the mapping

$$
\hat{u} \rightarrow \sup _{\hat{v} \in V_{\widehat{T}}} \frac{\int_{\widehat{T}} \hat{u} \psi_{\widehat{T}} \hat{v}}{\|\hat{v}\|_{0, q ; \widehat{T}}}
$$

defines a norm on $V_{\widehat{T}}$. Since $\operatorname{dim} V_{\widehat{T}}<\infty$, there is a constant $\hat{c}>0$ such that

$$
\hat{c}\|\hat{u}\|_{0, p ; \widehat{T}} \leq \sup _{\hat{v} \in V_{\widehat{T}}} \frac{\int_{\widehat{T}} \hat{u} \psi_{\widehat{T}} \hat{v}}{\|\hat{v}\|_{0, q ; \widehat{T}}} \quad \forall \hat{u} \in V_{\widehat{T}} .
$$

Now, take an arbitrary $u \in V_{T}$. Set $\hat{u}:=u \circ F_{T} \in V_{\widehat{T}}$ and choose a $\hat{w} \in V_{\widehat{T}}$ such that

$$
\|\hat{w}\|_{0, q ; \widehat{T}}=1 \text { and } \int_{\widehat{T}} \hat{u} \psi_{\widehat{T}} \hat{w} \geq \hat{c}\|\hat{u}\|_{0, p ; \widehat{T}} .
$$

With $w:=\hat{w} \circ F_{T}^{-1}$ we then obtain

$$
\begin{aligned}
\sup _{v \in V_{T}} \frac{\int_{T} u \psi_{T} v}{\|v\|_{0, q ; T}} & \geq \frac{\int_{T} u \psi_{T} w}{\|w\|_{0, q ; T}}=\left|\operatorname{det} B_{T}\right|^{1-1 / q} \int_{\widehat{T}} \hat{u} \psi_{\widehat{T}} \hat{w} \\
& \geq \hat{c}\left|\operatorname{det} B_{T}\right|^{1 / p}\|\hat{u}\|_{0, p ; \widehat{T}}=\hat{c}\|u\|_{0, p ; T} .
\end{aligned}
$$

The proof of the lower bound of equation (5.4) is completely analogous. One only has to replace $\left|\operatorname{det} B_{T}\right|$ by $\beta_{T}$.

The mappings

$$
\hat{u} \rightarrow\left\|\nabla\left(\psi_{\widehat{T}} \hat{u}\right)\right\|_{0, q ; \widehat{T}} \text { and } \hat{\sigma} \rightarrow\left\|\nabla\left(\psi_{\widehat{E}} \hat{P} \hat{\sigma}\right)\right\|_{0, q ; \widehat{T}}
$$

define norms on $V_{\widehat{T}}$ and $V_{\widehat{E}}$. Since $\psi_{\widehat{T}}$ and $\psi_{\widehat{E}}$ vanish at the vertices of $\widehat{T}$, and since $\operatorname{dim} V_{\widehat{T}}<\infty$ and $\operatorname{dim} V_{\widehat{E}}<\infty$, these norms are equivalent to $\left\|\psi_{\widehat{T}} \hat{u}\right\|_{0, q ; \widehat{T}}$ and $\left\|\psi_{\widehat{E}} \hat{P} \hat{\sigma}\right\|_{0, q ; \widehat{T}}$, respectively. Estimates (5.5), (5.6) now follow in the usual way by transforming to $\widehat{T}$, using the equivalence of norms there, and transforming back to $T$.

With the same arguments as above we finally conclude that there is a constant $\tilde{c}>0$ such that

$$
\left\|\psi_{\widehat{E}} \widehat{P} \hat{\sigma}\right\|_{0, q ; \widehat{T}} \leq \tilde{c}\|\hat{\sigma}\|_{q ; \widehat{E}} \quad \forall \hat{\sigma} \in V_{\widehat{E}}
$$


Since

$$
\left|\operatorname{det} B_{T}\right| \leq\left|\left\|B _ { T } \left|\left\|^{n} \leq c h_{T}^{n}, \quad \beta_{T}^{-1} \leq\right\|\left\|B_{T}^{-1} \mid\right\|^{n-1} \leq c h_{T}^{1-n},\right.\right.\right.
$$

this implies for all $\sigma \in V_{E}$

$$
\begin{aligned}
\left\|\psi_{E} P \sigma\right\|_{0, q ; T} & =\left|\operatorname{det} B_{T}\right|^{1 / q}\left\|\psi_{\widehat{E}} \widehat{P} \hat{\sigma}\right\|_{0, q ; \widehat{T}} \leq \tilde{c}\left|\operatorname{det} B_{T}\right|^{1 / q}\|\hat{\sigma}\|_{q ; \widehat{E}} \\
& =\tilde{c}\left|\operatorname{det} B_{T}\right|^{1 / q} \beta_{T}^{-1 / q}\|\sigma\|_{q ; E} \leq c_{7} h_{T}^{1 / q}\|\sigma\|_{q ; E} .
\end{aligned}
$$

Remark 5.2. The estimates of Lemma 5.1 also hold for "slightly curved" simplices. More precisely, assume that the transformation $F_{T}$ is no longer affine, but that it still is a diffeomorphism. Let $A_{T}: \widehat{T} \rightarrow \mathbb{R}^{n}$ be the invertible affine mapping which is uniquely determined by the condition that $A_{T}^{-1} \circ F_{T}$ leaves the vertices of $\widehat{T}$ invariant. Denote by $\alpha_{T}$ the Gram determinant of the transformation of $\widehat{E}$ induced by $A_{T}$. A simple perturbation argument then shows that the estimates of Lemma 5.1 remain valid, provided

$$
\begin{gathered}
\|\| I-D F_{T}^{-1} D A_{T} \mid\|\|_{0, \infty ; \widehat{T}}, \quad\|\| I-D A_{T}^{-1} D F_{T}\|\|_{0, \infty ; \widehat{T}}, \\
\left\|1-\left|\operatorname{det} D F_{T}\right|^{-1}\left|\operatorname{det} D A_{T}\right|\right\|_{0, \infty ; \widehat{T}}, \quad\left\|1-\beta_{T}^{-1} \alpha_{T}\right\|_{0, \infty ; \widehat{T}}
\end{gathered}
$$

are smaller than a positive threshold which only depends on the constants in the corresponding estimates on $\widehat{T}$.

Thanks to Lemma 5.1, we may construct in the next section spaces $\tilde{Y}_{h}$ and $\widehat{Y}_{h}$ satisfying the conditions of Propositions 4.1 and 4.3 by considering all linear combinations of functions $\psi_{T} v$ and $\psi_{E} P \sigma$, where $v$ and $\sigma$ vary in suitable spaces $V_{T}$ and $V_{E}$, respectively, and $T$ and $E$ run through all simplices and faces of the finite element partition.

Note that Lemma 5.1 does not depend on the fact that $\psi_{\widehat{T}}$ and $\psi_{\widehat{E}}$ are polynomials. This special choice has only been made for convenience.

\section{SCAlAR QUASI-LINEAR ELLIPTIC EQUATIONS OF 2ND ORDER}

Consider the boundary value problem

$$
\begin{aligned}
-\nabla \cdot \underline{a}(x, u, \nabla u) & =b(x, u, \nabla u) \text { in } \Omega, \\
u & =0 \text { on } \Gamma,
\end{aligned}
$$

where $b \in C\left(\Omega \times \mathbb{R} \times \mathbb{R}^{n}, \mathbb{R}\right)$ and $\underline{a} \in C^{1}\left(\Omega \times \mathbb{R} \times \mathbb{R}^{n}, \mathbb{R}^{n}\right)$ are such that the matrix $A(x, y, z):=\left(\frac{1}{2}\left(\partial_{z_{j}} a_{i}(x, y, z)+\partial_{z_{i}} a_{j}(x, y, z)\right)_{1 \leq i, j \leq n}\right.$ is positive definite for all $x \in \Omega, y \in \mathbb{R}, z \in \mathbb{R}^{n}$.

Under suitable growth conditions on $\underline{a}, b$, and their derivatives there are real numbers $1<r, q<\infty$ such that the weak formulation of problem (6.1) fits into the framework of $\S 2$ with

$$
\begin{gathered}
X:=\left\{u \in W^{1, r}(\Omega): u=0 \text { on } \Gamma\right\}, \quad\|\cdot\|_{X}:=\|\cdot\|_{1, r}, \\
Y:=\left\{\varphi \in W^{1, q}(\Omega): u=0 \text { on } \Gamma\right\}, \quad\|\cdot\|_{Y}:=\|\cdot\|_{1, q}, \\
\langle F(u), \varphi\rangle:=\int_{\Omega} \underline{a}(x, u, \nabla u) \nabla \varphi-\int_{\Omega} b(x, u, \nabla u) \varphi .
\end{gathered}
$$

Denote by $p:=\frac{q}{q-1}$ the dual exponent of $q$. Note that $D F(u) \in \operatorname{Isom}\left(X, Y^{*}\right)$ if the linear boundary value problem

$$
\begin{gathered}
-\nabla \cdot(A(x, u, \nabla u) \nabla v)-\nabla \cdot\left(\partial_{y} \underline{a}(x, u, \nabla u) v\right) \\
-\nabla_{z} b(x, u, \nabla u) \cdot \nabla v-\partial_{y} b(x, u, \nabla u) v=f \text { in } \Omega, \\
v=0 \text { on } \Gamma
\end{gathered}
$$


admits for each $f \in Y^{*}$ a unique weak solution $v \in X$ which depends continuously on $f$.

Some examples of problems falling into the present category are given by:

(1) The equations of prescribed mean curvature:

$$
\begin{aligned}
\underline{a}(x, u, \nabla u) & :=\left[1+\|\nabla u\|^{2}\right]^{-1 / 2} \nabla u, \\
b(x, u, \nabla u) & :=f(x) \in L^{2}(\Omega), \\
r & :=q:=2 .
\end{aligned}
$$

(2) The $\alpha$-Laplacian:

$$
\begin{aligned}
\underline{a}(x, u, \nabla u) & :=\|\nabla u\|^{\alpha-2} \nabla u, \quad \alpha>1, \\
b(x, u, \nabla u) & :=f(x) \in L^{p}(\Omega), \\
r & :=q:=\alpha .
\end{aligned}
$$

(3) The subsonic flow of an irrotational, ideal, compressible gas:

$$
\begin{aligned}
\underline{a}(x, u, \nabla u) & :=\left[1-\frac{\gamma-1}{2}\|\nabla u\|^{2}\right]^{1 /(\gamma-1)} \nabla u, \quad \gamma>1, \\
b(x, u, \nabla u) & :=f(x) \in L^{p}(\Omega), \\
r & :=q:=\frac{2 \gamma}{\gamma-1} .
\end{aligned}
$$

(4) The stationary heat equation with convection and nonlinear diffusion coefficient:

$$
\begin{gathered}
\underline{a}(x, u, \nabla u):=k(u) \nabla u, \\
b(x, u, \nabla u):=f-\mathbf{c} \cdot \nabla u, \\
f \in L^{\infty}(\Omega), \quad \mathbf{c} \in C\left(\Omega, \mathbb{R}^{n}\right), \quad k \in C^{2}(\mathbb{R}), \\
k(s) \geq \alpha>0, \quad\left|k^{(l)}(s)\right| \leq \gamma, \quad \forall s \in \mathbb{R}, \quad l=0,1,2, \\
r:=p \in(2,4) .
\end{gathered}
$$

(5) Bratu's equation:

$$
\begin{aligned}
\underline{a}(x, u, \nabla u) & :=\nabla u, \\
b(x, u, \nabla u) & :=\lambda e^{u}, \quad \lambda>0, \\
r & :=p>n .
\end{aligned}
$$

(6) A nonlinear eigenvalue problem:

$$
\begin{aligned}
\underline{a}(x, u, \nabla u) & :=\nabla u, \\
b(x, u, \nabla u) & :=\lambda u-u^{\beta}, \quad \beta \geq n, \\
r & :=p \geq n .
\end{aligned}
$$

Example (2) fits into the framework of Proposition 2.1 if $\alpha \geq 2$. If $1<\alpha<$ 2 , the corresponding function $F$ is no longer differentiable. However, it still fits into the framework of Remark 2.3 with

$$
\varrho(t)=\underline{c}\left\{\left\|u_{0}\right\|_{X}+t\right\}^{\alpha-2} t, \quad \sigma(t)=\bar{c} t^{\alpha-1}
$$

(cf. $[10, \S 5.3])$. 
In example (5) there is a critical parameter $\lambda^{*}>0$ such that the problem admits two weak solutions if $0<\lambda<\lambda^{*}$, exactly one weak solution if $\lambda=\lambda^{*}$, and no solution if $\lambda>\lambda^{*}$. The solution corresponding to $\lambda=\lambda^{*}$ is a turning point and fits into the framework of the second part of $\S 3$ (cf. [12]).

Example (6) always admits the trivial solution. If $\lambda$ is a simple eigenvalue of the Laplacian, there is a simple bifurcation which fits into the framework of the third part of $\S 3$ (cf. [12]).

We do not specify the discretization of problem (6.1) in detail. We only assume that $X_{h} \subset X \cap W^{1, \infty}(\Omega)$ and $Y_{h} \subset Y \cap W^{1, \infty}(\Omega)$ are finite element spaces corresponding to $\mathscr{T}_{h}$ consisting of affinely equivalent elements in the sense of [10], and that $S_{h}^{1,0} \cap Y \subset Y_{h}$.

In order to construct $R_{h}, \widetilde{F}_{h}$ and $\widetilde{Y}_{h}$, we define two integers $k, l$ and approximations $\underline{a}_{h}$ of $\underline{a}$ and $b_{h}$ of $b$ as follows:

$$
\begin{aligned}
& \underline{a}_{h}\left(x, u_{h}, \nabla u_{h}\right):= \begin{cases}\underline{a}\left(x, u_{h}, \nabla u_{h}\right), & \text { if } \underline{a}\left(x, v_{h}, \nabla v_{h}\right) \in S_{h}^{k,-1} \forall v_{h} \in X_{h}, \\
\sum_{T \in \mathscr{T}_{h}} \pi_{1, T} a\left(x, u_{h}, \nabla u_{h}\right), k:=1, & \text { otherwise, }\end{cases} \\
& b_{h}\left(x, u_{h}, \nabla u_{h}\right):= \begin{cases}b\left(x, u_{h}, \nabla u_{h}\right), & \text { if } b\left(x, v_{h}, \nabla v_{h}\right) \in S_{h}^{l,-1} \forall v_{h} \in X_{h}, \\
\sum_{T \in \mathscr{T}_{h}} \pi_{0, T} b\left(x, u_{h}, \nabla u_{h}\right), l:=0 \text { otherwise. }\end{cases}
\end{aligned}
$$

Here, $u_{h} \in X_{h}$ is arbitrary. Now, $\widetilde{F}_{h}$ is defined in the same way as $F$ with $\underline{a}$ and $b$ replaced by $\underline{a}_{h}$ and $b_{h}$, respectively, $R_{h}:=I_{h}$, and

$$
\tilde{Y}_{h}:=\operatorname{span}\left\{\psi_{T} v, \psi_{E} P \sigma:\left.v \in \Pi_{m}\right|_{T},\left.\sigma \in \Pi_{k}\right|_{E}, T \in \mathscr{T}_{h}, E \in \mathscr{E}_{h, \Omega}\right\},
$$

where $m:=\max \{k-1, l\}$.

Put, for abbreviation,

$$
\begin{gathered}
\varepsilon_{T}:=\left\{h_{T}^{p} \|-\nabla \cdot\left(\underline{a}\left(\cdot, u_{h}, \nabla u_{h}\right)-\underline{a}_{h}\left(\cdot, u_{h}, \nabla u_{h}\right)\right)\right. \\
-\left(b\left(\cdot, u_{h}, \nabla u_{h}\right)-b_{h}\left(\cdot, u_{h}, \nabla u_{h}\right)\right) \|_{0, p ; T}^{p} \\
+\sum_{E \subset \partial T \backslash \Gamma} h_{E} \|\left[n \cdot \left(\underline{a}\left(\cdot, u_{h}, \nabla u_{h}\right)\right.\right. \\
\left.\left.\left.\quad-\underline{a}_{h}\left(\cdot, u_{h}, \nabla u_{h}\right)\right)\right]_{E} \|_{p ; E}^{p}\right\}^{1 / p} \forall T \in \mathscr{T}_{h},
\end{gathered}
$$

$$
\begin{aligned}
\eta_{T}:=\left\{h_{T}^{p} \|-\nabla\right. & \underline{a}_{h}\left(\cdot, u_{h}, \nabla u_{h}\right)-b_{h}\left(\cdot, u_{h}, \nabla u_{h}\right) \|_{0, p ; T}^{p} \\
& \left.+\sum_{E \subset \partial T \backslash \Gamma} h_{E}\left\|\left[n \cdot \underline{a}_{h}\left(\cdot, u_{h}, \nabla u_{h}\right)\right]_{E}\right\|_{p ; E}^{p}\right\}^{1 / p} \forall T \in \mathscr{T}_{h} .
\end{aligned}
$$

The quantity $\varepsilon_{T}$ obviously measures the quality of the approximation of $\underline{a}$ and $b$ by $\underline{a}_{h}$ and $b_{h}$, respectively, and can be estimated explicitly. Below, we will show that $\left\|\left(\operatorname{Id}_{Y}-R_{h}\right)^{*}\left[F\left(u_{h}\right)-\widetilde{F}_{h}\left(u_{h}\right)\right]\right\|_{Y^{*}}$ and $\left\|F\left(u_{h}\right)-\widetilde{F}_{h}\left(u_{h}\right)\right\|_{\widetilde{Y}_{h}^{*}}$ are bounded from above by $c\left\{\sum_{T \in \mathscr{T}_{h}} \varepsilon_{T}^{p}\right\}^{1 / p}$. 
Note that

$$
\varepsilon_{T}=h_{T}\left\|f-\pi_{0 ; T} f\right\|_{0, p ; T} \quad \forall T \in \mathscr{T}_{h},
$$

if $X_{h} \subset S_{h}^{1,0}$ in examples (1)-(3), that

$$
\varepsilon_{T} \leq c h_{T}^{2(1-n / p)}\left\|\nabla u_{h}\right\|_{0, p ; T} \quad \forall T \in \mathscr{T}_{h}
$$

if $X_{h} \subset S_{h}^{1,0}$ in example (4), that

$$
\varepsilon_{T} \leq c h_{T}^{2}\left\|\nabla u_{h}\right\|_{0, p ; T} \exp \left(\left\|u_{h}\right\|_{0, \infty ; T}\right) \quad \forall T \in \mathscr{T}_{h}
$$

if $X_{h}$ consists of piecewise polynomials in example (5), and that

$$
\varepsilon_{T}=0 \quad \forall T \in \mathscr{T}_{h}
$$

if $X_{h}$ consists of piecewise polynomials and $\beta \in \mathbb{N}$ in example (6).

Using integration by parts elementwise, we obtain for all $\varphi \in Y$

$$
\begin{aligned}
\left\langle F\left(u_{h}\right), \varphi\right\rangle= & \sum_{T \in \mathscr{S}_{h}} \int_{T}\left\{-\nabla \cdot \underline{a}\left(x, u_{h}, \nabla u_{h}\right)-b\left(x, u_{h}, \nabla u_{h}\right)\right\} \varphi \\
& +\sum_{E \in \mathscr{E}_{h, \Omega}} \int_{E}\left[n \cdot \underline{a}\left(x, u_{h}, \nabla u_{h}\right)\right]_{E} \varphi
\end{aligned}
$$

and

$$
\begin{aligned}
\left\langle\widetilde{F}_{h}\left(u_{h}\right), \varphi\right\rangle= & \sum_{T \in T_{h}} \int_{T}\left\{-\nabla \cdot \underline{a}_{h}\left(x, u_{h}, \nabla u_{h}\right)-b_{h}\left(x, u_{h}, \nabla u_{h}\right)\right\} \varphi \\
& +\sum_{E \in \mathscr{E}_{h, \Omega}} \int_{E}\left[n \cdot \underline{a}_{h}\left(x, u_{h}, \nabla u_{h}\right)\right]_{E} \varphi
\end{aligned}
$$

Lemma 5.1, inequalities (5.1), (5.2), the definition of $\widetilde{Y}_{h}$, and equalities (6.4), (6.5) then imply that

$$
\begin{aligned}
& \left\|\left(\operatorname{Id}_{Y}-R_{h}\right)^{*}\left[F\left(u_{h}\right)-\widetilde{F}_{h}\left(u_{h}\right)\right]\right\|_{Y^{*}} \\
& =\sup _{\substack{\varphi \in Y \\
\|\varphi\|_{Y}=1}} \sum_{T \in \mathscr{S}_{h}} \int_{T}\left\{-\nabla \cdot\left(\underline{a}\left(x, u_{h}, \nabla u_{h}\right)-\underline{a}_{h}\left(x, u_{h}, \nabla u_{h}\right)\right)\right. \\
& \left.\quad-\left(b\left(x, u_{h}, \nabla u_{h}\right)-b_{h}\left(x, u_{h}, \nabla u_{h}\right)\right)\right\}\left\{\varphi-I_{h} \varphi\right\} \\
& \quad+\sum_{E \in \mathscr{E}_{h, \Omega}} \int_{E}\left[n \cdot\left(\underline{a}\left(x, u_{h}, \nabla u_{h}\right)-\underline{a}_{h}\left(x, u_{h}, \nabla u_{h}\right)\right)\right]_{E}\left\{\varphi-I_{h} \varphi\right\} \\
& \leq c\left\{\sum_{T \in \mathscr{S}_{h}} \varepsilon_{T}^{p}\right\}^{1 / p}
\end{aligned}
$$


and

(6.7)

$$
\begin{aligned}
& \left\|F\left(u_{h}\right)-\widetilde{F}_{h}\left(u_{h}\right)\right\|_{\widetilde{Y}_{h}^{*}} \\
& =\sup _{\substack{\varphi_{h} \in \widetilde{Y}_{h} \\
\left\|\varphi_{h}\right\|_{Y}=1}} \sum_{T \in \mathscr{T}_{h}} \int_{T}\left\{-\nabla \cdot\left(\underline{a}\left(x, u_{h}, \nabla u_{h}\right)-\underline{a}_{h}\left(x, u_{h}, \nabla u_{h}\right)\right)\right. \\
& \quad+\sum_{E \in \mathscr{E}_{h, \Omega}} \int_{E}\left[n \cdot\left(\underline{a}\left(x, u_{h}, \nabla u_{h}\right)-\underline{a}_{h}\left(x, u_{h}, \nabla u_{h}\right)\right)\right]_{E} \varphi_{h} \\
& \leq c\left\{\sum_{T \in \mathscr{T}_{h}} \varepsilon_{T}^{p}\right\}^{1 / p} .
\end{aligned}
$$

Similarly, we obtain

(6.8)

$$
\begin{aligned}
& \left\|\left(\operatorname{Id}_{Y}-R_{h}\right)^{*} \widetilde{F}_{h}\left(u_{h}\right)\right\|_{Y^{*}} \\
& =\sup _{\substack{\varphi \in Y \\
\|\varphi\|_{Y}=1}} \sum_{T \in \mathscr{T}_{h}} \int_{T}\left\{-\nabla \cdot \underline{a}_{h}\left(x, u_{h}, \nabla u_{h}\right)-b_{h}\left(x, u_{h}, \nabla u_{h}\right)\right\}\left\{\varphi-I_{h} \varphi\right\} \\
& \quad+\sum_{E \in \mathscr{E}_{h, \Omega}} \int_{E}\left[n \cdot \underline{a}_{h}\left(x, u_{h}, \nabla u_{h}\right)\right]_{E}\left\{\varphi-I_{h} \varphi\right\} \\
& \leq c\left\{\sum_{T \in \mathscr{T}_{h}} \eta_{T}^{p}\right\}^{1 / p}
\end{aligned}
$$

and

(6.9)

$$
\begin{aligned}
\left\|\widetilde{F}_{h}\left(u_{h}\right)\right\|_{\widetilde{Y}_{h}^{*}}= & \sup _{\substack{\varphi_{h} \in \widetilde{Y}_{h} \\
\left\|\varphi_{h}\right\|_{Y}=1}} \sum_{T \in \mathscr{T}_{h}} \int_{T}\left\{-\nabla \cdot \underline{a}_{h}\left(x, u_{h}, \nabla u_{h}\right)-b_{h}\left(x, u_{h}, \nabla u_{h}\right)\right\} \varphi_{h} \\
& +\sum_{E \in \mathscr{E}_{h}, \Omega} \int_{E}\left[n \cdot \underline{a}_{h}\left(x, u_{h}, \nabla u_{h}\right)\right]_{E} \varphi_{h} \\
\leq c & \left\{\sum_{T \in \mathscr{T}_{h}} \eta_{T}^{p}\right\}^{1 / p} .
\end{aligned}
$$

In order to prove inequality (4.1), consider an arbitrary simplex $T \in \mathscr{T}_{h}$ and an arbitrary face $E \in \mathscr{E}_{h, \Omega}$ of $T$ and denote by $\tilde{Y}_{h} \mid \omega, \omega \in\left\{T, \omega_{E}, \omega_{T}\right\}$, the set of all functions $\varphi \in \widetilde{Y}_{h}$ with $\operatorname{supp}(\varphi) \subset \omega$. Lemma 5.1, equation (6.5), and the definition of $\tilde{Y}_{h}$ then yield 


$$
\begin{aligned}
& c_{1} c_{4}^{-1} h_{T}\left\|-\nabla \cdot \underline{a}_{h}\left(\cdot, u_{h}, \nabla u_{h}\right)-b_{h}\left(\cdot, u_{h}, \nabla u_{h}\right)\right\|_{0, p ; T} \\
& \leq \sup _{v \in \Pi_{m \mid T} \backslash\{0\}}\left\|\nabla\left(\psi_{T} v\right)\right\|_{0, q ; T}^{-1} \\
& \quad \cdot \int_{T}\left\{-\nabla \cdot \underline{a}_{h}\left(x, u_{h}, \nabla u_{h}\right)-b_{h}\left(x, u_{h}, \nabla u_{h}\right)\right\} \psi_{T} v \\
& =\sup _{v \in \Pi_{m \mid T} \backslash\{0\}}\left\|\nabla\left(\psi_{T} v\right)\right\|_{0, q ; T}^{-1}\left\langle\widetilde{F}_{h}\left(u_{h}\right), \psi_{T} v\right\rangle \\
& \leq \sup _{\substack{\varphi \in \widetilde{Y}_{h \mid T} \\
\|\varphi\|_{Y}=1}}\left\langle\widetilde{F}_{h}\left(u_{h}\right), \varphi\right\rangle
\end{aligned}
$$

and, using inequality (6.10),

(6.11)

$$
\begin{aligned}
& c_{2} c_{6}^{-1} c_{7}^{-1} h_{E}^{1 / p}\left\|\left[n \cdot \underline{a}_{h}\left(\cdot, u_{h}, \nabla u_{h}\right)\right]_{E}\right\|_{p ; E} \\
& \leq \sup _{\sigma \in \Pi_{k \mid E \backslash\{0\}}} c_{6}^{-1} c_{7}^{-1} h_{E}^{1 / p}\|\sigma\|_{q ; E}^{-1} \int_{E}\left[n \cdot \underline{a}_{h}\left(x, u_{h}, \nabla u_{h}\right)\right]_{E} \psi_{E} P \sigma \\
& =\sup _{\sigma \in \Pi_{k \mid E} \backslash\{0\}} c_{6}^{-1} c_{7}^{-1} h_{E}^{1 / p}\|\sigma\|_{q ; E}^{-1} \\
& \qquad \\
& \qquad\left\{\left\langle\widetilde{F}_{h}\left(u_{h}\right), \psi_{E} P \sigma\right\rangle\right. \\
& \left.\quad-\int_{\omega_{E}}\left\{-\nabla \cdot \underline{a}_{h}\left(x, u_{h}, \nabla u_{h}\right)-b_{h}\left(x, u_{h}, \nabla u_{h}\right)\right\} \psi_{E} P \sigma\right\} \\
& \leq \sup _{\substack{\varphi \in \widetilde{Y}_{h} \mid \omega_{E} \\
\|\varphi\|_{Y}=1}}\left\langle\widetilde{F}_{h}\left(u_{h}\right), \varphi\right\rangle \\
& \quad+c_{6}^{-1} h_{E}\left\|-\nabla \cdot \underline{a}_{h}\left(\cdot, u_{h}, \nabla u_{h}\right)-b_{h}\left(\cdot, u_{h}, \nabla u_{h}\right)\right\|_{0, p ; \omega_{E}} \\
& \leq \sup _{\substack{\varphi \in \widetilde{Y}_{h \mid \omega_{E}} \\
\|\varphi\|_{Y}=1}}\left\langle\widetilde{F}_{h}\left(u_{h}\right), \varphi\right\rangle .
\end{aligned}
$$

Inequalities (6.10) and (6.11) imply that

$$
\eta_{T} \leq c \sup _{\substack{\varphi \in \widetilde{Y}_{h} \mid \omega_{T} \\\|\varphi\|_{Y}=1}}\left\langle\widetilde{F}_{h}\left(u_{h}\right), \varphi\right\rangle
$$

and

$$
\left\{\sum_{T \in \mathscr{T}_{h}} \eta_{T}^{p}\right\}^{1 / p} \leq c\left\|\widetilde{F}_{h}\left(u_{h}\right)\right\|_{\widetilde{Y}_{h}^{*}}
$$

Inequalities (6.8) and (6.13), in particular, prove inequality (4.1).

Propositions 2.1 and 4.1 and inequalities (6.6), (6.7), (6.8), (6.9), (6.12), and (6.13) yield the following a posteriori error estimates for problem (6.1).

Proposition 6.1. Let $u \in X$ be a weak solution of problem (6.1) which is regular in the sense of Proposition 2.1, and let $u_{h} \in X_{h}$ be an approximate solution of 
tine corresponding discrete problem which is sufficiently close to $u$ in the sense of Proposition 2.1. Then the following a posteriori error estimates hold:

and

$$
\begin{aligned}
\left\|u-u_{h}\right\|_{1, r} \leq & c_{1}\left\{\sum_{T \in \mathscr{Y}_{h}} \eta_{T}^{p}\right\}^{1 / p}+c_{2}\left\{\sum_{T \in \mathscr{T}_{h}} \varepsilon_{T}^{p}\right\}^{1 / p} \\
& +c_{3}\left\|F\left(u_{h}\right)-F_{h}\left(u_{h}\right)\right\|_{Y_{h}^{*}}+c_{4}\left\|F_{h}\left(u_{h}\right)\right\|_{Y_{h}^{*}}
\end{aligned}
$$

$$
\eta_{T} \leq c_{5}\left\|u-u_{h}\right\|_{1, r ; \omega_{T}}+c_{6}\left\{\sum_{T^{\prime} \subset \omega_{T}} \varepsilon_{T^{\prime}}^{p}\right\}^{1 / p} \quad \forall T \in \mathscr{T}_{h} .
$$

Here, $\varepsilon_{T}$ and $\eta_{T}$ are given by equations (6.2) and (6.3), and $\left\|F\left(u_{h}\right)-F_{h}\left(u_{h}\right)\right\|_{Y_{h}^{*}}$ and $\left\|F_{h}\left(u_{h}\right)\right\|_{Y_{h}^{*}}$ are the consistency error of the discretization and the residual of the discrete problem, respectively.

Remark 6.2. Proposition 6.1 can easily be extended to the case of Neumann boundary conditions. One only has to replace $\Gamma$ in equations (6.2) and (6.3) by the part of the boundary on which Dirichlet boundary conditions are imposed. The first estimate of Proposition 6.1 also holds if $\eta_{T}$ is defined using the original functions $\underline{a}$ and $b$ instead of the projected ones $\underline{a}_{h}$ and $b_{h}$. The $\varepsilon_{T}$-term then of course disappears. If the functions $a$ and $b$ are sufficiently smooth, one may also use higher-order approximations $\underline{a}_{h}$ and $b_{h}$ instead of the present low-order ones.

As mentioned before, Proposition 6.1 can be applied to example (2) only in the case $\alpha \geq 2$. Observing that for $1<\alpha<2$ the strong monotonicity of $F$ implies the unique solvability of the corresponding weak problem, we obtain from Remark 2.3 and inequalities (6.6), (6.7), (6.8), (6.9), and (6.13) the following result which complements the results of [9].

Proposition 6.3. Let $1<\alpha<2$ and denote by $u \in W^{1, \alpha}(\Omega), u=0$ on $\Gamma$, the unique solution of

$$
\int_{\Omega}\|\nabla u\|^{\alpha-2} \nabla u \nabla v=\int_{\Omega} f v \quad \forall v \in W^{1, \alpha}(\Omega), v=0 \text { on } \Gamma .
$$

Let $u_{h} \in X_{h}$ be an approximate solution of a discretization of the above problem. Then the following a posteriori error estimates hold:

$$
\begin{aligned}
\left\|u-u_{h}\right\|_{1, \alpha} \leq & c_{1}\left\{\sum_{T \in \mathscr{Y}_{h}} \eta_{T}^{\alpha}\right\}^{1 / \alpha}+c_{2}\left\{\sum_{T \in \mathscr{Y}_{h}} \varepsilon_{T}^{\alpha}\right\}^{1 / \alpha} \\
& +c_{3}\left\|F\left(u_{h}\right)-F_{h}\left(u_{h}\right)\right\|_{Y_{h}^{*}}+c_{4}\left\|F_{h}\left(u_{h}\right)\right\|_{Y_{h}^{*}}
\end{aligned}
$$

and

$$
\left\{\sum_{T \in \mathscr{T}_{h}} \eta_{T}^{\alpha}\right\}^{1 / \alpha} \leq c_{5}\left\|u-u_{h}\right\|_{1, \alpha}^{1 /(\alpha-1)}+c_{6}\left\{\sum_{T \in \mathscr{T}_{h}} \varepsilon_{T}^{\alpha}\right\}^{1 / \alpha(\alpha-1)} .
$$

Here, $\varepsilon_{T}, \eta_{T},\left\|F\left(u_{h}\right)-F_{h}\left(u_{h}\right)\right\|_{Y_{h}^{*}}$, and $\left\|F_{h}\left(u_{h}\right)\right\|_{Y_{h}^{*}}$ are as in Proposition 6.1. Moreover,

$$
\varepsilon_{T}=h_{T}\left\|f-\Pi_{0, T} f\right\|_{0, \alpha ; T} \quad \forall T \in \mathscr{T}_{h},
$$

if $u_{h}$ is piecewise linear. 
As mentioned before, example (6) exhibits a simple bifurcation from the trivial branch at the simple eigenvalues of the Laplacian. Combining the results of $\S 3$ with those of this section, we obtain the following a posteriori error estimate.

Proposition 6.4. Denote by $\lambda^{*} \in \mathbb{R}$ and $u^{*} \in W^{1, p}(\Omega), u^{*}=0$ on $\Gamma, p>n, a$ simple eigenvalue of the Laplace equation with homogeneous Dirichlet boundary conditions and a corresponding eigenfunction with $\int_{\Omega} u^{*}=1$. Let $\lambda_{h} \in \mathbb{R}$ and $u_{h} \in X_{h}$ be a solution of

$$
\int_{\Omega} \nabla u_{h} \nabla v_{h}-\lambda_{h} \int_{\Omega} u_{h} v_{h}+\int_{\Omega} u_{h}^{\beta} v_{h}=0 \quad \forall v_{h} \in X_{h},
$$

where $X_{h} \subset\left\{v \in W^{1, p}(\Omega) \cap W^{1, \infty}(\Omega): v=0\right.$ on $\left.\Gamma\right\}$ is a finite element space corresponding to $\mathscr{T}_{h}$ consisting of piecewise polynomials, and where $\beta \in \mathbb{N}$, $\beta \geq n$. If $\lambda_{h}$ and $u_{h}$ are sufficiently close to $\lambda^{*}$ and $u^{*}$, the following $a$ posteriori error estimates hold:

$$
\left|\lambda_{h}-\lambda^{*}\right|+\left\|u_{h}-u^{*}\right\|_{1, p} \leq c_{1}\left\{\left|\int_{\Omega} u_{h}-1\right|+\left\{\sum_{T \in \mathscr{T}_{h}} \eta_{T}^{p}\right\}^{1 / p}\right\}
$$

and

$$
\left|\int_{\Omega} u_{h}-1\right|+\left\{\sum_{T \in \mathscr{T}_{h}} \eta_{T}^{p}\right\}^{1 / p} \leq c_{2}\left\{\left|\lambda_{h}-\lambda^{*}\right|+\left\|u_{h}-u^{*}\right\|_{1, p}\right\}
$$

where

$$
\eta_{T}:=\left\{h_{T}^{p}\left\|-\Delta u_{h}-\lambda_{h} u_{h}\right\|_{0, p ; T}^{p}+\sum_{E \subset \partial T \backslash \Gamma} h_{E}\left\|\left[\partial_{n} u_{h}\right]_{E}\right\|_{p ; E}^{p}\right\}^{1 / p} .
$$

Proof. Observe that the consistency error of the above discretization vanishes; Proposition 6.4 then follows from inequalities (6.6), (6.7), (6.8), and (6.9) and the results of the third part of $\S 3$ with $l \in \mathscr{L}(V, \mathbb{R})$ given by

$$
l(v):=\int_{\Omega} v \quad \forall v \in W^{1, p}(\Omega), v=0 \text { on } \Gamma .
$$

When comparing Propositions 6.1 and 6.4, we remark that the latter only yields global lower bounds on the error. This is due to the global nature of the functional $l$ defined above.

We conclude this section with a simple example of an a posteriori error estimator which is based on the solution of auxiliary local problems and which generalizes the estimator introduced in $[4,5]$. For simplicity we assume that $p=q=r=2$. We choose an arbitrary vertex $x_{0}$ in the partition $\mathscr{T}_{h}$ and keep it fixed in what follows. Denote by $\mathscr{T}_{0}$ and $\mathscr{E}_{0}$ the set of all $T \in \mathscr{T}_{h}$ and of all $E \in \mathscr{E}_{h}$, respectively, which have $x_{0}$ as a vertex. Put $\omega_{0}:=\bigcup_{T \in \mathscr{F}_{0}} T$. Let

$$
\widehat{X}_{h}:=\widehat{Y}_{h}:=\left.\tilde{Y}_{h}\right|_{\omega_{0}},
$$

and define the operator $B \in \mathscr{L}\left(\widehat{X}_{h}, \widehat{Y}_{h}^{*}\right)$ by

$$
\langle B u, \varphi\rangle:=\int_{\omega_{0}} \nabla \varphi^{t} A_{0} \nabla u \quad \forall u \in \widehat{X}_{h}, \varphi \in \widehat{Y}_{h},
$$


where

$$
A_{0}:=A\left(x_{0}, u_{h}\left(x_{0}\right), \pi_{0, \omega_{0}}\left(\nabla u_{h}\right)\right) .
$$

Note that the operator $B$ is obtained by first linearizing around $u_{h}$ the differential operator associated with problem (6.1), then freezing the coefficients of the resulting linear operator at $x_{0}$, and finally retaining only the principal part of the linear constant-coefficient operator. Since $\nabla u_{h}$ may be discontinuous, its value at $x_{0}$ is approximated by the $L^{2}$-projection $\pi_{0, \omega_{0}}\left(\nabla u_{h}\right)$. Other constructions are of course also possible.

Since the matrix $A(x, y, z)$ is symmetric and positive definite for all $x \in$ $\Omega, y \in \mathbb{R}, z \in \mathbb{R}^{n}$, and since the functions in $\widehat{X}_{h}=\widehat{Y}_{h}$ vanish on $\partial \omega_{0}$, we immediately obtain from Korn's inequality that $B \in \operatorname{Isom}\left(\widehat{X}_{h}, \widehat{Y}_{h}^{*}\right)$. Let $u_{0} \in \widehat{X}_{h}$ be the unique solution of

$$
\left\langle B u_{0}, \psi\right\rangle=\left\langle\widetilde{F}_{h}\left(u_{h}\right), \varphi\right\rangle \quad \forall \varphi \in \widehat{Y}_{h},
$$

and set

$$
\eta_{x_{0}}:=\left\|u_{0}\right\|_{1,2 ; \omega_{0}} .
$$

Note that problem $(6.14)$ is equivalent to

$$
\int_{\omega_{0}} \nabla \varphi^{t} A_{0} \nabla u_{0}=\int_{\omega_{0}} \underline{a}_{h}\left(x, u_{h}, \nabla u_{h}\right) \nabla \varphi-\int_{\omega_{0}} b_{h}\left(x, u_{h}, \nabla u_{h}\right) \varphi \quad \forall \varphi \in \widehat{Y}_{h} .
$$

This shows that $\eta_{x_{0}}$ falls into the class of a posteriori error estimators originally introduced in $[4,5]$ for the Poisson equation.

Lemma 5.1 and equations (6.5) and (6.12) immediately imply that

$$
\underline{c}\left\|\widetilde{F}_{h}\left(u_{h}\right)\right\|_{\widehat{Y}_{h}} \leq\left\{\sum_{T \in \mathscr{T}_{0}} \eta_{T}^{2}\right\}^{1 / 2} \leq \bar{c}\left\|\widetilde{F}_{h}\left(u_{h}\right)\right\|_{\widehat{Y}_{h}}
$$

Together with Proposition 4.3, this yields the following result.

Proposition 6.5. Let $x_{0}$ be an arbitrary vertex in the triangulation $\mathscr{T}_{h}$. Then there are two constants $c_{1}, c_{2}$, which only depend on the polynomial degree of the space $X_{h}$ and on the ratio $h_{T} \varrho_{T}$, such that the following inequalities hold:

$$
c_{1}\left\{\sum_{T \in \mathscr{T}_{0}} \eta_{T}^{2}\right\}^{1 / 2} \leq \eta_{x_{0}} \leq c_{2}\left\{\sum_{T \in \mathscr{T}_{0}} \eta_{T}^{2}\right\}^{1 / 2} .
$$

Here, $\eta_{T}$ and $\eta_{x_{0}}$ are given by equations (6.3) and (6.15), respectively.

\section{EIGENVALUE PROBLEMS FOR SCALAR LINEAR ELLIPTIC OPERATORS OF 2ND ORDER}

As an example for the treatment of eigenvalue problems, we consider in this section the problem

$$
\begin{aligned}
-\nabla \cdot(A(x) \nabla u)+d(x) u & =\lambda u & & \text { in } \Omega, \\
u & =0 & & \text { on } \Gamma .
\end{aligned}
$$

Here, $d \in C\left(\Omega, \mathbb{R}_{+}\right)$and $A \in C^{1}\left(\Omega, \mathbb{R}^{n \times n}\right)$ are such that $A$ is symmetric and uniformly positive definite on $\Omega$. Of course, we are only interested in solutions $u$ which do not identically vanish on $\Omega$. 
When considering $\lambda$ as a parameter, problem (7.1) can be treated as a bifurcation problem similar to example (6) of the previous section. Here, we adopt a different strategy and define

$$
\begin{aligned}
& X:=Y:=\mathbb{R} \times\left\{u \in W^{1,2}(\Omega): u=0 \text { on } \Gamma\right\} \\
&\|\cdot\|_{X}:=\|\cdot\|_{Y}:=\left\{\mid \cdot \dot{i}^{2}+\|\cdot\|_{1,2}^{2}\right\}^{1 / 2} \\
&\langle F([\lambda, u]),[\mu, v]\rangle:=\int_{\Omega}\left\{\nabla v^{t} A \nabla u+d u v-\lambda u v\right\}+\mu\left\{\int_{\Omega} u^{2}-1\right\} .
\end{aligned}
$$

Then, $[\lambda, u] \in X,\|u\|_{0,2}=1$, is a weak solution of problem (7.1) if and only if it is a solution of equation (1.1). Moreover, one easily checks that $[\lambda, u]$ is a regular solution in the sense of Proposition 2.1 if and only if $\lambda$ is a simple eigenvalue of the differential operator associated with problem (7.1).

As in the previous section, we do not specify the discretization of problem (7.1) in detail. We only assume that

$$
\begin{gathered}
X_{h}=\mathbb{R} \times V_{h} \subset X, \quad Y_{h}=\mathbb{R} \times W_{h} \subset Y, \\
\left\langle F_{h}\left(\left[\lambda_{h}, u_{h}\right]\right),\left[\mu_{h}, v_{h}\right]\right\rangle=\left\langle F\left(\left[\lambda_{h}, u_{h}\right]\right),\left[\mu_{h}, v_{h}\right]\right\rangle \\
\forall\left[\lambda_{h}, u_{h}\right] \in X_{h},\left[\mu_{h}, v_{h}\right] \in Y_{h},
\end{gathered}
$$

where $V_{h}, W_{h}$ are finite element spaces corresponding to $\mathscr{T}_{h}$ which consist of affinely equivalent elements in the sense of [10] and which satisfy $\left\{v_{h} \in\right.$ $S_{h}^{1,0}: v_{h}=0$ on $\left.\Gamma\right\} \subset W_{h}$. Obviously, the consistency error of the above discretization vanishes. Moreover, $\left[\lambda_{h}, u_{h}\right] \in X_{h}$ is a solution of equation (1.2) if and only if

$$
\begin{gathered}
\int_{\Omega}\left\{\nabla v_{h}^{t} A \nabla u_{h}+d u_{h} v_{h}\right\}=\lambda_{h} \int_{\Omega} u_{h} v_{h} \quad \forall v_{h} \in W_{h}, \\
\int_{\Omega} u_{h}^{2}=1 .
\end{gathered}
$$

Hence, problem (1.2) is equivalent to a standard finite-dimensional eigenvalue problem. In what follows, we will always assume that $\left[\lambda_{h}, u_{h}\right] \in X_{h}$ is a solution of problem (7.2).

Let $m$ be the maximal polynomial degree of the functions in $W_{h}$. Proceeding as in the previous section, we set

$$
\begin{aligned}
A_{h} & :=\sum_{T \in \mathscr{T}_{h}} \pi_{1, T} A, \\
d_{h} & :=\sum_{T \in \mathscr{T}_{h}} \pi_{0, T} d, \\
R_{h} & :=\left[0, I_{h}\right], \\
\tilde{Y}_{h} & :=\mathbb{R} \times \operatorname{span}\left\{\psi_{T} v, \psi_{E} P \sigma: v \in \Pi_{m}\left|T, \sigma \in \Pi_{m}\right|_{E}, T \in \mathscr{T}_{h}, E \in \mathscr{E}_{h, \Omega}\right\}, \\
\varepsilon_{T} & :=\left\{h_{T}^{2}\left\|-\nabla \cdot\left(\left(A-A_{h}\right) \nabla u_{h}\right)+\left(d-d_{h}\right) u_{h}\right\|_{0,2 ; T}^{2}\right. \\
& \left.\quad+\sum_{E \subset \partial T \backslash \Gamma} h_{E}\left\|\left[n \cdot\left(\left(A-A_{h}\right) \nabla u_{h}\right)\right]_{E}\right\|_{2 ; E}^{2}\right\}^{1 / 2},
\end{aligned}
$$




$$
\begin{aligned}
\eta_{T}:=\left\{h_{T}^{2} \|-\nabla\right. & \cdot\left(A_{h} \nabla u_{h}\right)+d_{h} u_{h}-\lambda_{h} u_{h} \|_{0,2 ; T}^{2} \\
& \left.+\sum_{E \subset \partial T \backslash \Gamma} h_{E}\left\|\left[n \cdot\left(A_{h} \nabla u_{h}\right)\right]_{E}\right\|_{2 ; E}^{2}\right\}^{1 / 2},
\end{aligned}
$$

and define $\widetilde{F}_{h}$ in the same way as $F$ with $A$ and $d$ replaced by $A_{h}$ and $d_{h}$, respectively. Note that

$$
\varepsilon_{T} \leq c h_{T}^{2}\left\{\|A\|_{2, \infty ; T}\left\|u_{h}\right\|_{1,2 ; T}+\|d\|_{1, \infty ; T}\left\|u_{h}\right\|_{0,2 ; T}\right\} .
$$

With the same arguments as in the previous section we conclude that

$$
\begin{aligned}
\left\langle F\left(\left[\lambda_{h}, u_{h}\right]\right),[\mu, v]\right\rangle= & \sum_{T \in \mathscr{F}_{h}} \int_{T}\left\{-\nabla \cdot\left(A \nabla u_{h}\right)+d u_{h}-\lambda_{h} u_{h}\right\} v \\
& +\sum_{E \in \mathscr{E}_{h, \Omega}} \int_{E}\left[n \cdot\left(A \nabla u_{h}\right)\right]_{E} v \quad \forall[\mu, v] \in Y, \\
\left\langle\widetilde{F}_{h}\left(\left[\lambda_{h}, u_{h}\right]\right),[\mu, v]\right)= & \sum_{T \in \mathscr{T}_{h}} \int_{T}\left\{-\nabla \cdot\left(A_{h} \nabla u_{h}\right)+d_{h} u_{h}-\lambda_{h} u_{h}\right\} v \\
& +\sum_{E \in \mathscr{E}_{h, \Omega}} \int_{E}\left[n \cdot\left(A_{h} \nabla u_{h}\right)\right]_{E} v \quad \forall[\mu, v] \in Y,
\end{aligned}
$$

$$
\begin{gathered}
\left\|\left(\operatorname{Id}_{Y}-R_{h}\right)^{*}\left[F\left(\left[\lambda_{h}, u_{h}\right]\right)-\widetilde{F}_{h}\left(\left[\lambda_{h}, u_{h}\right]\right)\right]\right\|_{Y^{*}} \leq c\left\{\sum_{T \in \mathscr{T}_{h}} \varepsilon_{T}^{2}\right\}^{1 / 2}, \\
\left\|F\left(\left[\lambda_{h}, u_{h}\right]\right)-\widetilde{F}_{h}\left(\left[\lambda_{h}, u_{h}\right]\right)\right\|_{\widetilde{Y}_{h}^{*}} \leq c\left\{\sum_{T \in \mathscr{T}_{h}} \varepsilon_{T}^{2}\right\}^{1 / 2}, \\
\left\|\left(\mathrm{Id}_{Y}-R_{h}\right)^{*} \widetilde{F}_{h}\left(\left[\lambda_{h}, u_{h}\right]\right)\right\|_{Y^{*}} \leq c\left\{\sum_{T \in \mathscr{T}_{h}} \eta_{T}^{2}\right\}^{1 / 2}, \\
\left\|\widetilde{F}_{h}\left(\left[\lambda_{h}, u_{h}\right]\right)\right\|_{\widetilde{Y}_{h}^{*}} \leq c\left\{\sum_{T \in \mathscr{T}_{h}} \eta_{T}^{2}\right\}^{1 / 2}, \\
\eta_{T} \leq \sup _{\substack{[0, v] \in \widetilde{Y}_{h} \\
\operatorname{supp} v \subset \omega_{T}}}^{\|[0, v]\|_{Y}^{-1}\left\langle\widetilde{F}_{h}\left(\left[\lambda_{h}, u_{h}\right]\right),[0, v]\right\rangle .}
\end{gathered}
$$

Inequalities (7.8) and (7.10), in particular, prove inequality (4.1).

Propositions 2.1 and 4.1 and inequalities (7.6)-(7.10) yield the following a posteriori error estimate for problem (7.1).

Proposition 7.1. Let $\lambda$ be a simple eigenvalue of the differential operator associated with problem (7.1), and let $u$ be a corresponding eigenfunction with 
$\|u\|_{0,2}=1$. Let $\left[\lambda_{h}, u_{h}\right] \in X_{h}$ be a solution of problem (7.2) which is sufficiently close to $[\lambda, u]$ in the sense of Proposition 2.1. Then the following a posteriori error estimates hold:

$$
\left|\lambda-\lambda_{h}\right|+\left\|u-u_{h}\right\|_{1,2} \leq c_{1}\left\{\sum_{T \in \mathscr{T}_{h}} \eta_{T}^{2}\right\}^{1 / 2}+c_{2}\left\{\sum_{T \in \mathscr{T}_{h}} \varepsilon_{T}^{2}\right\}^{1 / 2}
$$

and

$$
\left\{\sum_{T \in \mathscr{T}_{h}} \eta_{T}^{2}\right\}^{1 / 2} \leq c_{3}\left\{\left|\lambda-\lambda_{h}\right|+\left\|u-u_{h}\right\|_{1,2}\right\}+c_{4}\left\{\sum_{T \in \mathscr{T}_{h}} \varepsilon_{T}^{2}\right\}^{1 / 2},
$$

where the constants $c_{1}, \ldots, c_{4}$ only depend on the polynomial degree of the spaces $V_{h}$ and $W_{h}$ and on the ratio $h_{T} / \varrho_{T}$, and where $\varepsilon_{T}$ and $\eta_{T}$ are given by equations (7.3) and (7.4), respectively.

Remark 7.2. The condition that $\left[\lambda_{h}, u_{h}\right]$ has to be sufficiently close to $[\lambda, u]$ essentially means that $\left|\lambda-\lambda_{h}\right|$ has to be smaller than the distance of $\lambda$ to its neighboring eigenvalues. In contrast to Proposition 6.1, we obtain in Proposition 7.1 only a global lower bound on the error. This is due to the global nature of the constraint $\int_{\Omega} u^{2}=1$ inherent in the definition of $F$. Proposition 7.1 can easily be extended to the case of Neumann boundary conditions. One only has to replace $\Gamma$ in equations (7.3) and (7.4) by the part of the boundary on which Dirichlet boundary conditions are imposed.

\section{StATIONARY, INCOMPRESSible NAVIER-STOKes EQUATIONS}

As an example for the treatment of elliptic systems we consider the stationary, incompressible Navier-Stokes equations

$$
\begin{aligned}
-\nu \Delta \mathbf{u}+(\mathbf{u} \cdot \nabla) \mathbf{u}+\nabla p & =\mathbf{f} & & \text { in } \Omega, \\
\nabla \cdot \mathbf{u} & =0 & & \text { in } \Omega, \\
\mathbf{u} & =0 & & \text { on } \Gamma,
\end{aligned}
$$

where $\nu>0$ is the constant viscosity of the fluid.

In order to cast problem (8.1) into the framework of $\S 2$, set

$$
M:=\left\{\mathbf{u} \in W^{1,2}(\Omega)^{n}: \mathbf{u}=0 \text { on } \Gamma\right\}, \quad Q:=\left\{p \in L^{2}(\Omega): \int_{\Omega} p=0\right\},
$$

and define

$$
\begin{gathered}
X:=Y:=M \times Q, \quad\|\cdot\|_{X}:=\|\cdot\|_{Y}:=\left\{\|\cdot\|_{1,2}^{2}+\|\cdot\|_{0,2}^{2}\right\}^{1 / 2} \\
\langle F([\mathbf{u}, p]),[\mathbf{v}, q]\rangle:=\nu \int_{\Omega} \nabla \mathbf{u} \nabla \mathbf{v}+\int_{\Omega}(\mathbf{u} \cdot \nabla) \mathbf{u v}-\int_{\Omega} p \nabla \cdot \mathbf{v}+\int_{\Omega} q \nabla \cdot \mathbf{u}-\int_{\Omega} \mathbf{f v} .
\end{gathered}
$$

Let $M_{h} \subset M$ and $Q_{h} \subset Q$ be two finite element spaces corresponding to $\mathscr{T}_{h}$ consisting of affinely equivalent elements in the sense of [10]. We assume that there are two integers $k, l \geq 1$ such that

$$
\left[S_{h}^{1,0}\right]^{n} \cap M \subset M_{h} \subset\left[S_{h}^{k, 0}\right]^{n}
$$

and

$$
S_{h}^{1,0} \cap Q \subset Q_{h} \subset S_{h}^{l, 0} \quad \text { or } \quad S_{h}^{0,-1} \cap Q \subset Q_{h} \subset S_{h}^{l,-1} \text {. }
$$


Define

$$
X_{h}:=Y_{h}:=M_{h} \times Q_{h},
$$

$$
\begin{aligned}
\left\langle F_{h}\left(\left[\mathbf{u}_{h}, p_{h}\right]\right),\left[\mathbf{v}_{h}, q_{h}\right]\right\rangle \\
:=\left\langle F\left(\left[\mathbf{u}_{h}, p_{h}\right]\right),\left[\mathbf{v}_{h}, q_{h}\right]\right\rangle \\
\quad+\delta \sum_{T \in \mathscr{T}_{h}} h_{T}^{2} \int_{T}\left\{-\nu \Delta \mathbf{u}_{h}+\left(\mathbf{u}_{h} \cdot \nabla\right) \mathbf{u}_{h}+\nabla p_{h}-\mathbf{f}\right\}\left\{\left(\mathbf{u}_{h} \cdot \nabla\right) \mathbf{v}_{h}+\nabla q_{h}\right\} \\
\quad+\delta \sum_{E \in \mathscr{C}_{h, \Omega}} h_{E} \int_{E}\left[p_{h}\right]_{E}\left[q_{h}\right]_{E}+\alpha \delta \int_{\Omega} \nabla \cdot \mathbf{u}_{h} \nabla \cdot \mathbf{v}_{h} .
\end{aligned}
$$

Here, $\alpha \geq 0, \delta \geq 0$ are stability parameters. If $\alpha>0, \delta>0$, the above discretization is capable of stabilizing both the influence of the convection term and of the divergence constraint without any conditions about the spaces $M_{h}$, $Q_{h}$ or the Peclet number $h_{T} \nu^{-1}$ (cf. [23], where also optimal a priori error estimates are established). The case $\alpha=\delta=0$ corresponds to the standard mixed finite element discretization of problem (8.1). The spaces $M_{h}, Q_{h}$ then have to satisfy the Babuška-Brezzi condition

$$
\inf _{p_{h} \in Q_{h} \backslash\{0\}} \sup _{\mathbf{u}_{h} \in M_{h} \backslash\{0\}} \frac{\int_{T} p_{h} \nabla \cdot \mathbf{u}_{h}}{\left\|p_{h}\right\|_{0,2}\left\|\mathbf{u}_{h}\right\|_{1,2}} \geq \beta>0
$$

with a constant $\beta$ independent of $h$. Moreover, the Peclet number $h_{T} \nu^{-1}$ must be sufficiently small in order to balance the influence of the convection term (cf. [17], where examples of spaces $M_{h}, Q_{h}$ satisfying inequality (8.3) are also given).

If $\alpha=\delta=0$, the consistency error obviously vanishes. If $\delta>0$, we conclude from standard inverse estimates that it is bounded by

$$
\begin{aligned}
& \left\|F\left(\left[\mathbf{u}_{h}, p_{h}\right]\right)-F_{h}\left(\left[\mathbf{u}_{h}, p_{h}\right]\right)\right\|_{Y_{h}^{*}} \\
& =\sup _{\substack{\left[\mathbf{v}_{h}, q_{h}\right] \in Y_{h} \\
\left\|\left[\mathbf{v}_{h}, q_{h}\right]\right\|_{Y}=1}} \delta \sum_{T \in \mathscr{T}_{h}} h_{T}^{2} \int_{T}\left\{-\nu \Delta \mathbf{u}_{h}+\left(\mathbf{u}_{h} \cdot \nabla\right) \mathbf{u}_{h}+\nabla p_{h}-\mathbf{f}\right\} \\
& \cdot\left\{\left(\mathbf{u}_{h} \cdot \nabla\right) \mathbf{v}_{h}+\nabla q_{h}\right\} \\
& +\delta \sum_{E \in \mathscr{E}_{h}, \Omega} h_{E} \int_{E}\left[p_{h}\right]_{E}\left[q_{h}\right]_{E}+\alpha \delta \int_{\Omega} \nabla \cdot \mathbf{u}_{h} \nabla \cdot \mathbf{v}_{h} \\
& \leq c(1+\alpha) \delta\left(1+\left\|\mathbf{u}_{h}\right\|_{1,2}\right) \\
& \cdot\left\{\sum_{T \in \mathscr{T}_{h}}\left[h_{T}^{2}\left\|-\nu \Delta \mathbf{u}_{h}+\left(\mathbf{u}_{h} \cdot \nabla\right) \mathbf{u}_{h}+\nabla p_{h}-\mathbf{f}\right\|_{0,2 ; T}^{2}+\left\|\nabla \cdot \mathbf{u}_{h}\right\|_{0,2 ; T}^{2}\right]\right. \\
& \left.+\sum_{E \in \mathscr{E}_{h, \Omega}} h_{E}\left\|\left[p_{h}\right]_{E}\right\|_{2 ; E}^{2}\right\}^{1 / 2}
\end{aligned}
$$

In order to cast this discretization into the framework of $\S 4$, we define $\widetilde{F}_{h}$ in 
the same way as $F$ with $\mathbf{f}$ replaced by $\pi_{0, T} \mathbf{f}$ and set

$$
\begin{gathered}
R_{h}[\mathbf{u}, p]:=\left[I_{h} u_{1}, \ldots, I_{h} u_{n}, 0\right], \\
\tilde{Y}_{h}:=\operatorname{span}\left\{\left[\psi_{T} \mathbf{v}, 0\right],\left[\psi_{E} P \sigma, 0\right],\left[0, \psi_{T} p\right]: \mathbf{v} \in\left[\Pi_{m} \mid T\right]^{n}, \sigma \in\left[\Pi_{m^{\prime}} \mid E\right]^{n},\right. \\
\left.p \in \Pi_{k-1} \mid T, T \in \mathscr{T}_{h}, E \in \mathscr{E}_{h, \Omega}\right\},
\end{gathered}
$$

where $m:=\max \{2 k-1, l-1\}$ and $m^{\prime}:=\max \{k-1, l\}$.

Lemma 5.1 and inequality (5.1) immediately imply

$$
\begin{aligned}
\left\|\widetilde{F}_{h}\left(\left[\mathbf{u}_{h}, p_{h}\right]\right)-F\left(\left[\mathbf{u}_{h}, p_{h}\right]\right)\right\|_{\widetilde{Y}_{h}^{*}} & =\sup _{\substack{\left[\mathbf{v}_{h}, q_{h}\right] \in \widetilde{Y}_{h} \\
\left\|\left[\mathbf{v}_{h}, q_{h}\right]\right\|_{Y}=1}} \sum_{T \in \mathscr{T}_{h}} \int_{T}\left(\mathbf{f}-\pi_{0, T} \mathbf{f}\right) \mathbf{v}_{h} \\
& \leq c\left\{\sum_{T \in \mathscr{F}_{h}} h_{T}^{2}\left\|\mathbf{f}-\pi_{0, T} \mathbf{f}\right\|_{0,2 ; T}^{2}\right\}^{1 / 2}
\end{aligned}
$$

and

$$
\begin{aligned}
\|\left(\mathbf{I d}_{Y}-R_{h}\right)^{*}\left[\widetilde{F}_{h}\left(\left[\mathbf{u}_{h}, p_{h}\right]\right)-F\left(\left[\mathbf{u}_{h}, p_{h}\right]\right) \|_{Y^{*}}\right. \\
=\sup _{\substack{[\mathbf{v}, q] \in Y \\
\| \mathbf{v}, q] \|_{Y}=1}} \sum_{T \in \mathscr{T}_{h}} \sum_{i=1}^{n} \int_{T}\left(f_{i}-\pi_{0, T} f_{i}\right)\left(v_{i}-I_{h} v_{i}\right) \\
\leq c\left\{\sum_{T \in \mathscr{T}_{h}} h_{T}^{2}\left\|\mathbf{f}-\pi_{0, T} \mathbf{f}\right\|_{0,2 ; T}^{2}\right\}^{1 / 2} .
\end{aligned}
$$

For abbreviation, we define for all $T \in \mathscr{T}_{h}$

$$
\begin{aligned}
\eta_{T}:=\left\{h_{T}^{2} \|\right. & -\nu \Delta \mathbf{u}_{h}+\left(\mathbf{u}_{h} \cdot \nabla\right) \mathbf{u}_{h}+\nabla p_{h}-\pi_{0, T} \mathbf{f} \|_{0,2 ; T}^{2} \\
& \left.+\sum_{E \subset \partial T \backslash \Gamma} h_{E}\left\|\left[\nu \partial_{n} \mathbf{u}_{h}-p_{h} \mathbf{n}\right]_{E}\right\|_{2 ; E}^{2}+\left\|\nabla \cdot \mathbf{u}_{h}\right\|_{0,2 ; T}^{2}\right\}^{1 / 2} .
\end{aligned}
$$

Observing that the identity

$$
\begin{aligned}
\left\langle\widetilde{F}_{h}\left(\left[\mathbf{u}_{h}, p_{h}\right]\right),[\mathbf{v}, q]\right\rangle & \\
= & \sum_{T \in \mathscr{T}_{h}}\left\{\int_{T}\left\{-\nu \Delta \mathbf{u}_{h}+\left(\mathbf{u}_{h} \cdot \nabla\right) \mathbf{u}_{h}+\nabla p_{h}-\pi_{0, T} \mathbf{f}\right\} \mathbf{v}+\int_{T} q \nabla \cdot \mathbf{u}_{h}\right\} \\
& +\sum_{E \in \mathscr{E}_{h, \Omega}} \int_{E}\left[\nu \partial_{h} \mathbf{u}_{h}-p_{h} \mathbf{n}\right]_{E} \mathbf{v}
\end{aligned}
$$


holds for all $[\mathbf{v}, q] \in Y$, we conclude from Lemma 5.1 and inequalities (5.1), (5.2) that

$$
\begin{aligned}
& \left\|\widetilde{F}_{h}\left(\left[\mathbf{u}_{h}, p_{h}\right]\right)\right\|_{\widetilde{Y}_{h}^{*}} \\
& \quad \sup _{\substack{\left[\mathbf{v}_{h}, q_{h}\right] \in \widetilde{Y}_{h} \\
\left\|\left[\mathbf{v}_{h}, q_{h}\right]\right\|_{Y}=1}} \sum_{T \in \mathscr{T}_{h}}\left\{\int_{T}\left\{-\nu \Delta \mathbf{u}_{h}+\left(\mathbf{u}_{h} \cdot \nabla\right) \mathbf{u}_{h}+\nabla p_{h}-\pi_{0, T} \mathbf{f}\right\} \mathbf{v}_{h}+\int_{T} q_{h} \nabla \cdot \mathbf{u}_{h}\right\} \\
& +\sum_{E \in \mathscr{E}_{h, \Omega}} \int_{E}\left[\nu \partial_{h} \mathbf{u}_{h}-p_{h} \mathbf{n}\right]_{E} \mathbf{v}_{h} \\
& \quad \leq c\left\{\sum_{T \in \mathscr{T}_{h}} \eta_{T}^{2}\right\}^{1 / 2}
\end{aligned}
$$

and

$$
\begin{aligned}
& \left\|\left(\mathbf{I d}_{Y}-R_{h}\right)^{*} \widetilde{F}_{h}\left(\left[\mathbf{u}_{h}, p_{h}\right]\right)\right\|_{Y^{*}} \\
& =\sup _{\substack{\| \mathbf{v}, q] \in Y \\
\|[\mathbf{v}, q]\|_{Y}=1}} \sum_{T \in \mathscr{S}_{h}}\left\{\sum_{i=1}^{n} \int_{T}\left\{-\nu \Delta u_{h, i}+\left(\mathbf{u}_{h} \cdot \nabla\right) u_{h, i}+\partial_{i} p_{h}-\pi_{0, T} f_{i}\right\}\right. \\
& \left.\cdot\left\{v_{i}-I_{h} v_{i}\right\}+\int_{T} q \nabla \cdot u_{h}\right\} \\
& +\sum_{E \in \mathscr{E}_{h, \Omega}} \sum_{i=1}^{n} \int_{E}\left[\nu \partial_{h} u_{h, i}-p_{h} n_{i}\right]_{E}\left(v_{i}-I_{h} v_{i}\right) \\
& \leq c\left\{\sum_{T \in \mathscr{S}_{h}} \eta_{T}^{2}\right\}^{1 / 2} .
\end{aligned}
$$

In order to prove inequality (4.1), consider an arbitrary simplex $T \in \mathscr{T}_{h}$ and an arbitrary face $E \in \mathscr{E}_{h, \Omega}$ of $T$ and define $\left.\widetilde{Y}_{h}\right|_{\omega}, \omega \in\left\{T, \omega_{E}, \omega_{T}\right\}$, as in $\S 6$. The definition of $\widetilde{Y}_{h}$, equation (8.8), and Lemma 5.1 then yield the estimates

$$
\begin{aligned}
c_{1} \| \nabla & \cdot \mathbf{u}_{h} \|_{0,2 ; T} \\
& \leq \sup _{r \in \Pi_{k-1} \mid r \backslash\{0\}}\|r\|_{0,2 ; T}^{-1} \int_{T} \nabla \cdot u_{h} \psi_{T} r \\
& =\sup _{r \in \Pi_{r-1} \mid T \backslash\{0\}}\left\langle\widetilde{F}_{h}\left(\left[\mathbf{u}_{h}, p_{h}\right]\right),\left[0, \psi_{T} r\right]\right\rangle\|r\|_{0,2 ; T}^{-1} \\
& \leq \sup _{\substack{[\mathbf{v}, q] \in \widetilde{Y}_{h \mid T} \\
\|[\mathbf{v}, q]\|}}\left\langle\widetilde{F}_{h}\left(\left[\mathbf{u}_{h}, p_{h}\right]\right),[\mathbf{v}, q]\right\rangle,
\end{aligned}
$$




$$
\begin{aligned}
c_{1} c_{4}^{-1} & h_{T}\left\|-\nu \Delta \mathbf{u}_{h}+\left(\mathbf{u}_{h} \cdot \nabla\right) \mathbf{u}_{h}+\nabla p_{h}-\pi_{0, T} \mathbf{f}\right\|_{0,2 ; T} \\
& \leq \sup _{\mathbf{w} \in\left[\Pi_{m} \mid T\right]^{n} \backslash\{0\}}\left\|\nabla\left(\psi_{T} \mathbf{w}\right)\right\|_{0,2 ; T}^{-1} \int_{T}\left\{-\nu \Delta \mathbf{u}_{h}+\left(\mathbf{u}_{h} \cdot \nabla\right) \mathbf{u}_{h}+\nabla p_{h}-\pi_{0, T} \mathbf{f}\right\} \psi_{T} \mathbf{w} \\
& =\sup _{\mathbf{w} \in\left[\Pi_{m} \mid T\right]^{n} \backslash\{0\}}\left\|\nabla\left(\psi_{T} \mathbf{w}\right)\right\|_{0,2 ; T}^{-1}\left\langle\widetilde{F}_{h}\left(\left[\mathbf{u}_{h}, p_{h}\right]\right),\left[\psi_{T} \mathbf{w}, 0\right]\right\rangle \\
& \leq \sup _{\substack{[\mathbf{v}, q] \in \widetilde{Y}_{h} \mid T \\
\|[\mathbf{v}, q]\| Y}}\left\langle\widetilde{F}_{h}\left(\left[\mathbf{u}_{h}, p_{h}\right],[\mathbf{v}, q]\right\rangle\right.
\end{aligned}
$$

and, using inequality (8.12),

$$
\begin{aligned}
& c_{2} c_{6}^{-1} c_{7}^{-1} h_{E}^{1 / 2}\left\|\left[\nu \partial_{h} \mathbf{u}_{h}-p_{h} \mathbf{n}\right]_{E}\right\|_{2 ; E} \\
& \leq \sup _{\sigma \in\left[\Pi_{m^{\prime}} \mid\right]^{n} \backslash\{0\}} c_{2} c_{6}^{-1} c_{7}^{-1} h_{E}^{1 / 2}\|\sigma\|_{2 ; E}^{-1} \int_{E}\left[\nu \partial_{n} \mathbf{u}_{h}-p_{h} \mathbf{n}\right] \psi_{E} P \sigma \\
& =\sup _{\sigma \in\left[\Pi_{m^{\prime}} \mid E\right]^{n} \backslash\{0\}} c_{2} c_{6}^{-1} c_{7}^{-1} h_{E}^{1 / 2}\|\sigma\|_{2 ; E}^{-1} \\
& \cdot\left\{\left\langle\widetilde{F}_{h}\left(\left[\mathbf{u}_{h}, p_{h}\right]\right),\left[\psi_{E} P \sigma, 0\right]\right\rangle\right. \\
& \left.-\int_{\omega_{E}}\left\{-\nu \Delta \mathbf{u}_{h}+\left(\mathbf{u}_{h} \cdot \nabla\right) \mathbf{u}_{h}+\nabla p_{h}-\pi_{0, T} \mathbf{f}\right\} \psi_{E} P \sigma\right\} \\
& \leq \sup _{\substack{\left.[\mathbf{v}, q] \in \widetilde{Y}_{h}\right|_{\omega_{E}} \\
\|[\mathbf{v}, q]\| \gamma=1}}\left\langle\widetilde{F}_{h}\left(\left[\mathbf{u}_{h}, p_{h}\right]\right),[\mathbf{v}, q]\right\rangle \\
& +c_{6}^{-1} h_{E}\left\|-\nu \Delta \mathbf{u}_{h}+\left(\mathbf{u}_{h} \cdot \nabla\right) \mathbf{u}_{h}+\nabla p_{h}-\pi_{0, T} \mathbf{f}\right\|_{0,2 ; \omega_{E}} \\
& \leq c \sup _{\substack{\left.[\mathbf{v}, q] \in \widetilde{Y}_{h}\right|_{\omega_{E}} \\
\|[\mathbf{v}, q]\|}}\left\langle\widetilde{F}_{h}\left(\left[\mathbf{u}_{h}, p_{h}\right]\right),[\mathbf{v}, q]\right\rangle .
\end{aligned}
$$

Inequalities (8.11)-(8.13) imply

$$
\eta_{T} \leq c \sup _{\substack{\left.[\mathbf{v}, q] \in \widetilde{Y}_{h}\right|_{\omega_{T}} \\\|[\mathbf{v}, q]\|_{Y}=1}}\left\langle\widetilde{F}_{h}\left(\left[\mathbf{u}_{h}, p_{h}\right]\right),[\mathbf{v}, q]\right\rangle
$$

and

$$
\left\{\sum_{T \in \mathscr{S}_{h}} \eta_{T}^{2}\right\}^{1 / 2} \leq c\left\|\widetilde{F}_{h}\left(\left[\mathbf{u}_{h}, p_{h}\right]\right)\right\|_{\widetilde{Y}_{h}^{*}} .
$$

Inequalities (8.9), (8.10), and (8.15) prove inequality (4.1) and show that, up to multiplicative constants, $\left\|\widetilde{F}_{h}\left(\left[\mathbf{u}_{h}, p_{h}\right]\right)\right\|_{\widetilde{Y}_{h}^{*}}$ is bounded from above and from below by $\left\{\sum_{T} \eta_{T}^{2}\right\}^{1 / 2}$. Propositions 2.1 and 4.1 and inequalities (8.4), (8.5), (8.6), (8.9), and (8.14) now yield the following a posteriori error estimate, which is a generalization of the results in [25, 27]. 
Proposition 8.1. Let $[\mathbf{u}, p]$ be a weak solution of problem (8.1) which is regular in the sense of Proposition 2.1, and let $\left[\mathbf{u}_{h}, p_{h}\right] \in X_{h}$ be a solution of

$$
\left\langle F_{h}\left(\left[\mathbf{u}_{h}, p_{h}\right]\right),\left[\mathbf{v}_{h}, q_{h}\right]\right\rangle=0, \quad \forall\left[\mathbf{v}_{h}, q_{h}\right] \in Y_{h},
$$

where $F_{h}$ is given in equation (8.2), which is sufficiently close to $[\mathbf{u}, p]$ in the sense of Proposition 2.1. Then the following a posteriori error estimates hold:

$$
\begin{gathered}
\left\{\left\|\mathbf{u}-\mathbf{u}_{h}\right\|_{1,2}^{2}+\left\|p-p_{h}\right\|_{0,2}^{2}\right\}^{1 / 2} \leq c_{1}\left[1+(1+\alpha) \delta\left(1+\left\|\mathbf{u}_{h}\right\|_{1,2}\right)\right]\left\{\sum_{T \in \mathscr{T}_{h}} \eta_{T}^{2}\right\}^{1 / 2} \\
+c_{2}\left\{\sum_{T \in \mathscr{T}_{h}} h_{T}^{2}\left\|\mathbf{f}-\pi_{0, T} \mathbf{f}\right\|_{0,2 ; T}^{2}\right\}^{1 / 2}, \\
\eta_{T} \leq c_{3}\left\{\left\|\mathbf{u}-\mathbf{u}_{h}\right\|_{1,2 ; \omega_{T}}^{2}+\left\|p-p_{h}\right\|_{0,2 ; \omega_{T}}^{2}\right\}^{1 / 2} \\
+c_{4}\left\{\sum_{T^{\prime} \subset \omega_{T}} h_{T^{\prime}}^{2}\left\|\mathbf{f}-\pi_{0, T^{\prime}} \mathbf{f}\right\|_{0,2 ; T^{\prime}}^{2}\right\}^{1 / 2},
\end{gathered}
$$

where $\eta_{T}$ is given by equation (8.7) and the constants $c_{1}, \ldots, c_{4}$ only depend on the polynomial degrees of the spaces $M_{h}, Q_{h}$ and on the ratio $h_{T} / \varrho_{T}$.

Remark 8.2. Proposition 8.1 can be extended to the case of the slip boundary condition

$$
\mathbf{u} \cdot \mathbf{n}=\mathbf{T}(\nu \mathbf{u}, p)-[\mathbf{n} \cdot \mathbf{T}(\nu \mathbf{u}, p) \cdot \mathbf{n}] \mathbf{n}=0,
$$

where

$$
\mathbf{T}(\mathbf{u}, p):=\left(\frac{1}{2}\left(\partial_{i} u_{j}+\partial_{j} u_{i}\right)-p \delta_{i j}\right)_{1 \leq i, j \leq n}
$$

denotes the stress tensor. One then has to replace $\nu \nabla \mathbf{u}-p \mathbf{I}$ in equation (8.7) by $\mathbf{T}(\nu \mathbf{u}, p)$, and $\Gamma$ by the part of the boundary on which the no-slip condition $\mathbf{u}=$ 0 is imposed. Here, $\mathbf{I}:=\left(\delta_{i j}\right)_{1 \leq i, j \leq n}$ denotes the unit tensor. Of course, the discretization then also has to take account of the different boundary condition (cf., e.g., $[24,26])$.

Remark 8.3. The previous results can also be extended to non-Newtonian fluids. Combining the arguments used to establish Propositions 6.1, 6.3, and 8.1, one can prove that the error estimator of [9] also yields local lower bounds similar to the second estimate of Proposition 8.1.

Next, we introduce an a posteriori error estimator for problem (8.1), which is based on the solution of discrete local Stokes problems and which fits into the framework of Proposition 4.3. This estimator is an extension to the NavierStokes equations of the one introduced in $[4,5]$ for the Poisson equation.

We choose an arbitrary vertex $x_{0}$ in the partition $\mathscr{T}_{h}$ and keep it fixed in what follows. Let $\omega_{0}, \mathscr{T}_{0}$ and $\mathscr{E}_{0}$ be as in $\S 6$. Put

$$
\begin{aligned}
M_{0} & :=\operatorname{span}\left\{\psi_{T} \mathbf{v}, \psi_{E} P \sigma: \mathbf{v} \in\left[\left.\Pi_{m^{\prime \prime}}\right|_{T}\right]^{n}, \sigma \in\left[\left.\Pi_{m^{\prime}}\right|_{E}\right]^{n}, T \in \mathscr{T}_{0}, E \in \mathscr{E}_{0}\right\}, \\
Q_{0} & :=\operatorname{span}\left\{\psi_{T} p: p \in \Pi_{k-1} \mid T, T \in \mathscr{T}_{0}\right\},
\end{aligned}
$$


where $m:=\max \{2 k-1, l-1\}, \quad m^{\prime}:=\max \{k-1, l\}$, and $m^{\prime \prime}:=$ $\max \{m, k+n-1\}$, and define

$$
\begin{aligned}
& \widehat{X}_{h}:=\widehat{Y}_{h}:=M_{0} \times Q_{0}, \\
&\langle B([\mathbf{v}, q]),[\mathbf{w}, r]\rangle:= \nu \int_{\omega_{0}} \nabla \mathbf{v} \nabla \mathbf{w}-\int_{\omega_{0}} q \nabla \cdot \mathbf{w} \\
&+\int_{\omega_{0}} r \nabla \cdot \mathbf{v} \quad \forall[\mathbf{v}, q],[\mathbf{w}, r] \in \widehat{X}_{h} .
\end{aligned}
$$

The definition of $m^{\prime \prime}$ implies that $\psi_{T} \nabla q \in M_{0}$ for all $q \in Q_{0}$. Together with Lemma 5.1, this shows that the spaces $M_{0}, Q_{0}$ satisfy an analogon of equation (8.3). Hence, we have $B \in \operatorname{Isom}\left(\widehat{X}_{h}, \widehat{Y}_{h}^{*}\right)$. Let $\left[\mathbf{u}_{0}, p_{0}\right] \in \widehat{X}_{h}$ be the unique solution of

$$
\left\langle B\left(\left[\mathbf{u}_{0}, p_{0}\right]\right),[\mathbf{w}, r]\right\rangle=\left\langle\widetilde{F}_{h}\left(\left[\mathbf{u}_{h}, p_{h}\right]\right),[\mathbf{w}, r]\right\rangle \quad \forall[\mathbf{w}, r] \in \tilde{Y}_{h},
$$

and define

$$
\eta_{x_{0}}:=\left\{\nu\left\|\mathbf{u}_{0}\right\|_{1,2 ; \omega_{0}}^{2}+\left\|p_{0}\right\|_{0,2 ; \omega_{0}}^{2}\right\}^{1 / 2} .
$$

Note, that problem $(8.16)$ is equivalent to

$$
\begin{aligned}
\nu \int_{\omega_{0}} \nabla \mathbf{u}_{0} \nabla \mathbf{w}-\int_{\omega_{0}} p_{0} \nabla \cdot \mathbf{w}= & \nu \int_{\omega_{0}} \nabla \mathbf{u}_{h} \nabla \mathbf{w}+\int_{\omega_{0}}\left(\mathbf{u}_{h} \cdot \nabla\right) \mathbf{u}_{h} \mathbf{w} \\
& -\int_{\omega_{0}} p_{h} \nabla \cdot \mathbf{w}-\int_{\omega_{0}} \pi_{0, T} \mathbf{f w} \quad \forall \mathbf{w} \in M_{0}, \\
\int_{\omega_{0}} r \nabla \cdot \mathbf{u}_{0}= & \int_{\omega_{0}} r \nabla \cdot \mathbf{u}_{h} \quad \forall r \in Q_{0} .
\end{aligned}
$$

This shows that $\eta_{x_{0}}$ falls into the class of a posteriori error estimators originally introduced in $[4,5]$ for the Poisson equation.

Obviously, we have $\left.\tilde{Y}_{h}\right|_{\omega_{0}} \subset \widehat{Y}_{h}$. Lemma 5.1 and equation (8.8), on the other hand, immediately imply

$$
\begin{aligned}
& \left\|\widetilde{F}_{h}\left(\left[\mathbf{u}_{h}, p_{h}\right]\right)\right\|_{\widehat{Y}_{h}^{*}} \\
& =\sup _{\substack{\left.[\mathbf{v}, q] \in \widehat{Y}_{h} \\
\| \mathbf{v}, q\right] \|_{Y}=1}} \sum_{T \in \mathscr{T}_{0}}\left\{\int_{T}\left\{-\nu \Delta \mathbf{u}_{h}+\left(\mathbf{u}_{h} \cdot \nabla\right) \mathbf{u}_{h}+\nabla p_{h}-\pi_{0, T} \mathbf{f}\right\} \mathbf{v}+\int_{T} q \nabla \cdot \mathbf{u}_{h}\right\} \\
& +\sum_{E \in \mathscr{E}_{0}} \int_{E}\left[\nu \partial_{h} \mathbf{u}_{h}-p_{h} \mathbf{n}\right]_{E} \mathbf{v} \\
& \leq c\left\{\sum_{T \in \mathscr{T}_{0}} \eta_{T}^{2}\right\}^{1 / 2}
\end{aligned}
$$

Together with inequality $(8.14)$, this proves

$$
\left\|\widetilde{F}_{h}\left(\left[\mathbf{u}_{h}, p_{h}\right]\right)\right\|_{\widehat{Y}_{h}^{*}} \leq c \sup _{\substack{[\mathbf{v}, q] \in \widetilde{Y}_{h \mid \omega_{0}} \\\|[\mathbf{v}, q]\|_{Y}=1}}\left\langle\widetilde{F}_{h}\left(\left[\mathbf{u}_{h}, p_{h}\right]\right),[\mathbf{v}, q]\right) .
$$

These results and Proposition 4.3 yield the following proposition, which is a generalization of results in $[4,5,25,27,28]$. 
Proposition 8.4. Let $x_{0}$ be an arbitrary vertex in the partition $\mathscr{T}_{h}$. Then there are two constants $c_{1}, c_{2}$, which only depend on the polynomial degree of the spaces $M_{h}, Q_{h}$ and on the ratio $h_{T} / \varrho_{T}$, such that the following inequalities hold:

$$
c_{1}\left\{\sum_{T \in \mathscr{O}_{0}} \eta_{T}^{2}\right\}^{1 / 2} \leq \eta_{x_{0}} \leq c_{2}\left\{\sum_{T \in \mathscr{T}_{0}} \eta_{T}^{2}\right\}^{1 / 2} .
$$

Here, $\eta_{T}$ and $\eta_{x_{0}}$ are given by equations (8.7) and (8.17), respectively.

\section{BIBLIOGRAPHY}

1. R. A. Adams, Sobolev spaces, Academic Press, New York, 1975.

2. I. Babuška, Feedback, adaptivity, and a posteriori estimates in finite elements: aims, theory, and experience, Accuracy Estimates and Adaptive Refinements in Finite Element Computation (I. Babuška et al., eds.), Wiley, New York, 1986, pp. 3-23.

3. I. Babuška and W. Gui, Basic principles of feedback and adaptive approaches in the finite element method, Comput. Methods Appl. Mech. Engrg. 55 (1986), 27-42.

4. I. Babuška and W. C. Rheinboldt, Error estimates for adaptive finite element computations, SIAM J. Numer. Anal. 15 (1978), 736-754.

5. Engrg. 12 (1978), 1597-1615.

6. I. Babuška and R. Rodriguez, The problem of the selection of an a-posteriori error indicator based on smoothing techniques, Internat. J. Numer. Methods. Engrg. (to appear).

7. R. E. Bank and A. Weiser, Some a posteriori error estimators for elliptic partial differential equations, Math. Comp. 44 (1985), 283-301.

8. R. E. Bank and D. B. Welfert, A posteriori error estimates for the Stokes equations: a comparison, Comput. Methods Appl. Mech. Engrg. 87 (1990), 323-340.

9. J. Baranger and H. El Amri, Estimateur a posteriori d'erreur pour le calcul adaptif d'écoulements quasi-Newtoniens, RAIRO Modél. Math. Anal. Numér. 25 (1991), 31-48.

10. Ph. G. Ciarlet, The finite element method for elliptic problems, North-Holland, Amsterdam, 1978.

11. Ph. Clément, Approximation by finite element functions using local regularization, RAIRO Modél. Math. Anal. Numér. 2 (1975), 77-84.

12. M. Crouzeix and J. Rappaz, On numerical approximation in bifurcation theory, Research in Appl. Math., vol. 13, Masson, Paris; Springer-Verlag, Berlin, 1990.

13. R. Duran, M. A. Muschietti, and R. Rodriguez, On the asymptotic exactness of error estimators for linear triangular elements, Numer. Math. 59 (1991), 107-127.

14. R. Duran and R. Rodriguez, On the asymptotic exactness of Bank-Weiser's estimator, Numer. Math. 62 (1992), 297-303.

15. K. Eriksson, Improved accuracy by adapted mesh-refinements in the finite element method, Math. Comp. 44 (1985), 321-343.

16. K. Eriksson and C. Johnson, An adaptive finite element method for linear elliptic problems, Math. Comp. 50 (1988), 361-383.

17. V. Girault and P.-A. Raviart, Finite element methods for Navier-Stokes equations: Theory and algorithms, Springer Ser. Comput. Math., vol. 5, Springer, Berlin, 1986.

18. J. T. Oden, L. Demkowicz, W. Rachowicz, and T. A. Westermann, Toward a universal $h-p$ adaptive finite element strategy, Part 2. A posteriori error estimation, Comput. Methods Appl. Mech. Engrg. 77 (1989), 113-180.

19. J. Pousin and J. Rappaz, Consistency, stability, a priori and a posteriori errors for PetrovGalerkin methods applied to nonlinear problems (Report), EPFL, Lausanne, 1992.

20. W. C. Rheinboldt, On a theory of mesh-refinement processes, SIAM J. Numer. Anal. 17 (1980), 766-778. 
21. T. Strouboulis and K. A. Hague, Recent experiences with error estimation and adaptivity I: Review of error estimators for scalar elliptic problems, Comput. Methods Appl. Mech. Engrg. 97 (1992), 399-436.

22. T. Strouboulis and J. T. Oden, A posteriori error estimation of finite element approximations in fluid mechanics, Comput. Methods Appl. Mech. Engrg. 78 (1990), 201-242.

23. L. Tobiska and R. Verfürth, Analysis of a streamline diffusion finite element method for the Stokes and Navier-Stokes equations (Report), Universities Magdeburg-Zürich, 1991.

24. R. Verfürth, Finite element approximation of incompressible Navier-Stokes equations with slip boundary conditions, Numer. Math. 50 (1987), 697-721.

25. _ A posteriori error estimators for the Stokes equations, Numer. Math. 55 (1989), 309-325.

26. __ Finite element approximation of incompressible Navier-Stokes equations with slip boundary conditions. II, Numer. Math. 59 (1991), 615-636.

27. __ A posteriori error estimators and adaptive mesh-refinement techniques for the NavierStokes equations, Incompressible CFD-Trends and Advances (M. D. Gunzburger and R. A. Nicolaides, eds.), Cambridge Univ Press, Cambridge, 1993, pp. 447-477.

28. _ A posteriori error estimation and adaptive mesh-refinement techniques, J. Comput. Appl. Math. (to appear).

29. J. Z. Zhu and O. C. Zienkiewicz, Adaptive techniques in the finite element method, Comm. Appl. Numer. Methods 4 (1988), 197-204.

30. O. C. Zienkiewicz and J. Z. Zhu, A simple error estimator and adaptive procedure for practical engineering analysis, Internat. J. Numer. Methods Engrg. 24 (1987), 337-357.

FAKUltät FÜr MATHEMATIK, RUhR-UNIVERSITÄt Bochum, D-44780 Bochum, GeRMANY 